\title{
Investigations of the output energy deviation and other parameters during commissioning of the four-rod radio frequency quadrupole at the Fermi National Accelerator Laboratory
}

\author{
J. S. Schmidt, ${ }^{*}$ B. Koubek, and A. Schempp \\ IAP, Goethe University, 60438 Frankfurt am Main, Germany \\ C. Y. Tan, D. S. Bollinger, K. L. Duel, P. R. Karns, W. A. Pellico, \\ V. E. Scarpine, and B. A. Schupbach \\ Fermi National Accelerator Laboratory, P.O. Box 500, Batavia, Illinois 60510-5011, USA \\ S. S. Kurennoy \\ Los Alamos National Laboratory, P.O. Box 1663, Los Alamos, New Mexico 87545, USA
}

(Received 23 October 2013; published 25 March 2014)

\begin{abstract}
After 30 years of operation, the Cockcroft-Walton based injector at the Fermi National Accelerator Laboratory has been replaced by a new beam line including a dimpled magnetron $35 \mathrm{keV}$ source in combination with a $750 \mathrm{keV}$ four-rod radio frequency quadrupole (RFQ). The new injector is followed by the existing drift tube linac. Prior to installation, a test beam line was built which included the magnetron source and the four-rod RFQ with a number of beam measurement instrumentation. The first beam test with the RFQ showed an output energy deviation greater than $2.5 \%$. Other problems also showed up which led to investigations of the output energy, power consumption and transmission properties using rf simulations which were complemented with additional beam measurements. The sources of this deviation and the mechanical modifications of the RFQ to solve this matter will be presented in this paper. Meanwhile, the nominal output energy of $750 \mathrm{keV}$ has been confirmed and the new injector with the four-rod RFQ is in full operation.
\end{abstract}

DOI: 10.1103/PhysRevSTAB.17.030102

PACS numbers: 29.20.Ej, 29.27.Ac

\section{THE INJECTOR UPGRADE AT FNAL}

The Cockcroft-Walton (C-W) injectors at FNAL (Fermi National Accelerator Laboratory) had been operational since 1978 and had been reliable sources of the $\mathrm{H}^{-}$beam for the FNAL program. However, after more than 30 years of operation, issues with maintenance, equipment obsolescence, and retirement of critical personnel had made continued reliable running of the $\mathrm{H}^{-}$injector increasingly more difficult.

An event that demonstrated the risk of the long term viability of the $\mathrm{C}-\mathrm{W}$ injector was the critical failure of the high voltage accelerating column of one of the C-Ws in June 2009 that led to 9 months of high energy physics operation without any redundancy. This failure clearly highlighted the vulnerability of the FNAL physics program, thus, FNAL management came to the conclusion that a replacement injector had to be found. The Proton Source (PS) group had recognized this problem earlier because at

\footnotetext{
schmidt@iap.uni-frankfurt.de

Published by the American Physical Society under the terms of the Creative Commons Attribution 3.0 License. Further distribution of this work must maintain attribution to the author $(s)$ and the published article's title, journal citation, and DOI.
}

the end of 2008 plans had already been started to design a new injector using a radio frequency quadrupole (RFQ). After the column failure, priority was given to the PS group to replace the C-Ws during the year-long 2011 shutdown.

As the PS group needed to make sure that the injector would not pose any major technical risks, it was decided that the new injector design would utilize the experience and design of the Brookhaven National Laboratory (BNL) proton injector. Similar projects such as the implementation of a RFQ with LINAC2 at CERN [1] have been done before and are well known. The BNL injector, like the FNAL injector, accelerates $\mathrm{H}^{-}$beam to $750 \mathrm{keV}$ before injection into the Linac and it runs at a rf of $201.25 \mathrm{MHz}$ which is the same as FNAL. Furthermore, the BNL injector has been working very reliably since its installation in 1988. Hence, critical parts of the design were chosen based on the BNL experience coupled with the following conservative choices: (i) Dimpled $\mathrm{H}^{-}$magnetron source: Comparable with the $\mathrm{H}^{-}$source presented by J. G. Allesi in [2], slit $\mathrm{H}^{-}$ magnetron sources have been used at FNAL for over 30 years and thus there is considerable experience and equipment on hand to make this a safe choice. (ii) Low energy beam transport (LEBT): A short, twin solenoid system was chosen because BNL had found that this design gave the best beam transport efficiency to the RFQ. (iii) RFQ: The 
TABLE I. Design Parameters of the FNAL RFQ.

\begin{tabular}{lcc}
\hline \hline Parameter & Design & Specifications \\
\hline Frequency & $201.25 \mathrm{MHz}$ & $201.25 \mathrm{MHz}$ \\
Input energy & $35 \mathrm{keV}$ & $35 \mathrm{keV}$ \\
Output energy & $753 \mathrm{keV}$ & $750 \mathrm{keV}$ \\
Beam current & $60 \mathrm{~mA}$ & $60 \mathrm{~mA}$ \\
Input transverse acceptance & $0.3 \pi \mathrm{mm}$ mrad (rms norm.) & $0.3 \pi \mathrm{mm} \mathrm{mrad} \mathrm{(rms} \mathrm{norm.)}$ \\
Output transverse emittance & $\epsilon_{x}=0.37 / \epsilon_{y}=0.35 \pi \mathrm{mm} \mathrm{mrad}$ & $>95 \%$ \\
Transmission efficiency & $98 \%$ & $100 \mathrm{~kW}$ beam power \\
Power consumption & & $0.12 \%$ \\
Duty factor & $1.18 \mathrm{~m}$ & \\
Electrode length & 102 & \\
Number of acc. cells & $4.17 \mathrm{~mm}$ & \\
Aperture (mean) & $0.3 \mathrm{~cm}$ & \\
Minimum radial aperture & $1 \leq m \leq 1.95$ & \\
Modulation & $72 \mathrm{kV}$ & \\
Intervane voltage & $25.18 \mathrm{MV} / \mathrm{m}$ & \\
Maximum peak surface field & & \\
\hline \hline
\end{tabular}

parameters that had been chosen for the RFQ are very conservative. See Table I. In fact, BNL has been operating a vane RFQ with similar parameters since 1988 and there has been very little downtime since its installation. (iv) Medium beam transport (MEBT): In principle, the RFQ can be installed right at the entrance of the first drift tube linac (DTL). For example at RAL [3]. However, the lattice of the FNAL DTL is not known precisely, and so a short MEBT that consists of two doublets and a buncher was designed to ensure that the beam can be matched into the DTL.

Taking all of the above into consideration, the final design of the injector is shown as a $3 \mathrm{D}$ CAD drawing in Fig. 1.

Despite best efforts by all parties concerned, problems cropped up in a few of the beamline components that had to be understood and fixed. Unfortunately, the RFQ had the most problems and they were severe enough that it

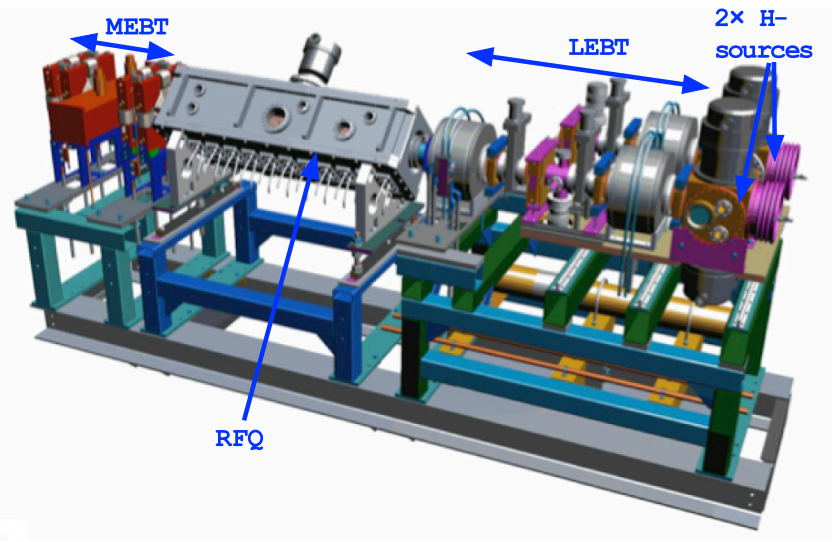

FIG. 1. A 3D CAD drawing of the new RFQ injector. There are two $\mathrm{H}^{-}$sources for redundancy, a short LEBT, RFQ, and a very short MEBT. could have sounded the death knell for the upgrade. Fortunately, after tremendous effort spent by the PS group, the manufacturer (IAP, Frankfurt) and colleagues at Los Alamos National Laboratory (LANL), most of the problems were understood, which then led to their resolution to a level that was deemed good enough to replace the C-Ws. The goal of this paper is to document the work that was done to identify, understand, and fix the problems with the RFQ and to show how design choices affect RFQ performance. Lessons learned here can only benefit the next generation of four-rod RFQs.

\section{DESIGN OF THE FOUR-ROD RFQ}

The key parameters of the FNAL injector are close to the $\mathrm{BNL} \mathrm{H}^{-}$injector setup. The design parameters of the RFQ are summarized in Table I together with the specifications defined by the FNAL PS group. These values were chosen because if the RFQ met them the new injector would maintain performance or even exceed the beam current and quality of the C-Ws [4].

The optimized design of the four-rod RFQ followed the principles described in [5] and yielded a considerably shorter RFQ with only 102 acceleration cells and an electrode length of $1.18 \mathrm{~m}$. In comparison, a plan based on the $\mathrm{BNL} \mathrm{H}^{-}$ injector assumed a length of $1.63 \mathrm{~m}$. For the acceleration of a $60 \mathrm{~mA}$ beam of $\mathrm{H}^{-}$ions from $35 \mathrm{keV}$ to $753 \mathrm{keV}$, a design transmission efficiency of $98 \%$ could be achieved.

The Parmteq $_{M}$ simulation results yield a transverse output rms emittance of $\epsilon_{x}=0.37 \pi \mathrm{mmmrad}$ and $\epsilon_{y}=0.35 \pi \mathrm{mm} \mathrm{mrad}$, and an unnormalized longitudinal emittance of $0.14 \mathrm{MeV}$ deg is based on an input distribution with a transverse rms emittance of $0.3 \pi \mathrm{mm}$ mrad in both planes. The particle dynamics design results are summerzied in Fig. 2. This vane design has been cross checked by G. Romanov [6], showing basically the same results. 

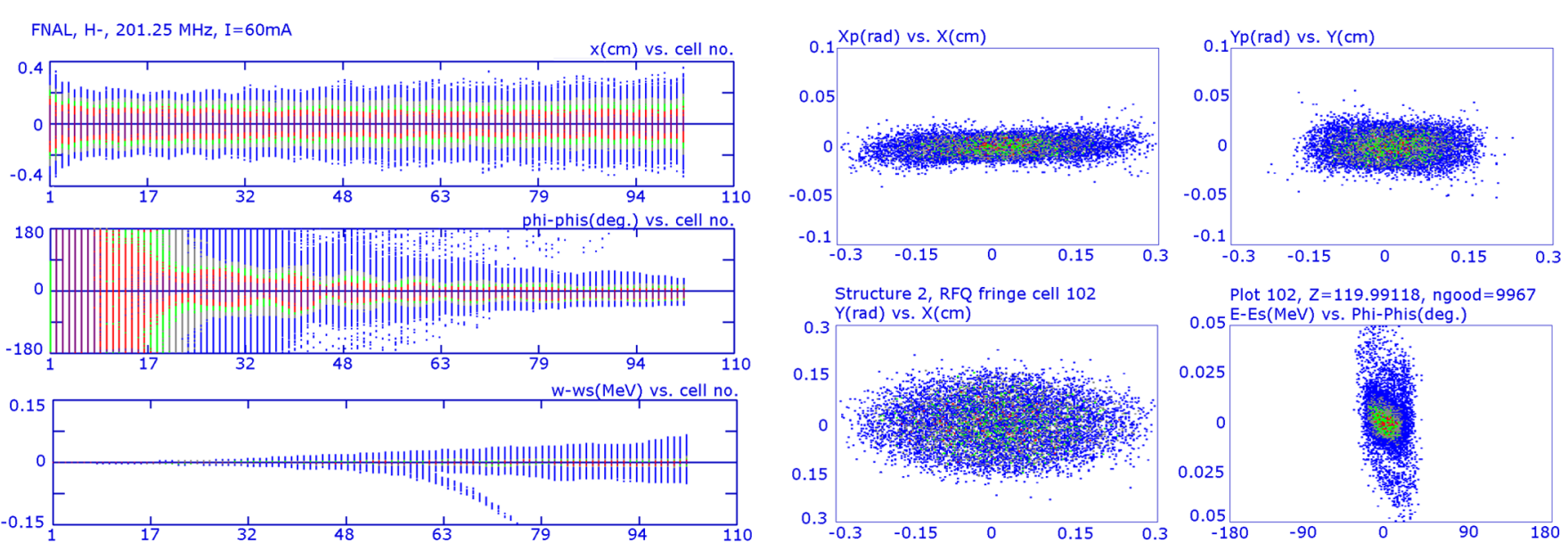

FIG. 2. Results of Parmteq $_{M}$ design. The calculated transmission is $98 \%$ with a transverse output rms emittance of $0.37 \pi$ mm mrad for $x$ and $0.35 \pi \mathrm{mm}$ mrad for $y$ and an unnormalized longitudinal emittance of $0.14 \mathrm{MeV} \mathrm{deg}$. In the right graphs the spatial distribution, the phase, and the energy evolution along the RFQ are pictured next to the output distributions in the left graphs.

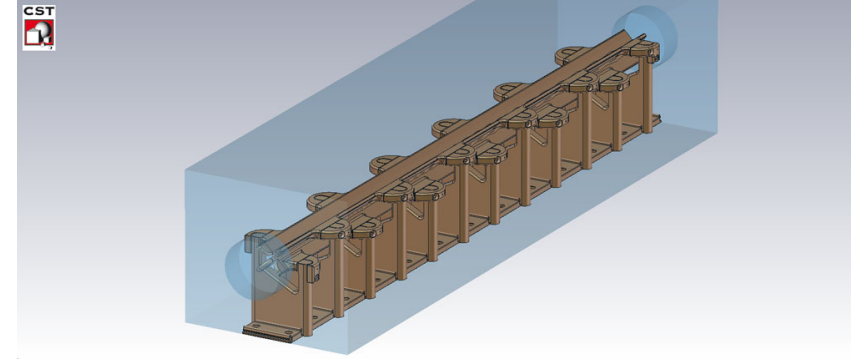

FIG. 3. CST MWS model of FNAL four-rod RFQ. The model includes the imported version of the original manufacture model with the modulated electrodes and the inside of the vacuum tank.

\section{A. $\mathrm{RF}$ characteristics}

A rf simulation model was built (see Fig. 3) with CST Microwave Studioß (MWS). Its geometric values with 11 rf cells and a beam height of $130 \mathrm{~mm}$ in the tank are summarized in Table II. In this definition, rf cell refers to the section of two stems with the part of the electrodes and the tuning plate in between. This model includes modulated electrodes which are completely identical to their manufacturing model for the $\mathrm{CNC}$ milling machine. Iterative steps with variations of the connections between the electrodes and stems or the electrodes shape were simulated to find a configuration to match the resonant frequency.

TABLE II. Geometric Parameter of the 4-Rod RFQ for FNAL.

\begin{tabular}{lc}
\hline \hline \# RF cells & 11 \\
Beam height in tank & $130 \mathrm{~mm}$ \\
Stem thickness & $20 \mathrm{~mm}$ \\
Stem distance (inner width) & $100 \mathrm{~mm}$ \\
Tank cross-section (inside) & $260 \mathrm{~mm}$ \\
Tank length & $1.29 \mathrm{~m}$ \\
Wall thickness & $45 \mathrm{~mm}$ \\
\hline \hline
\end{tabular}

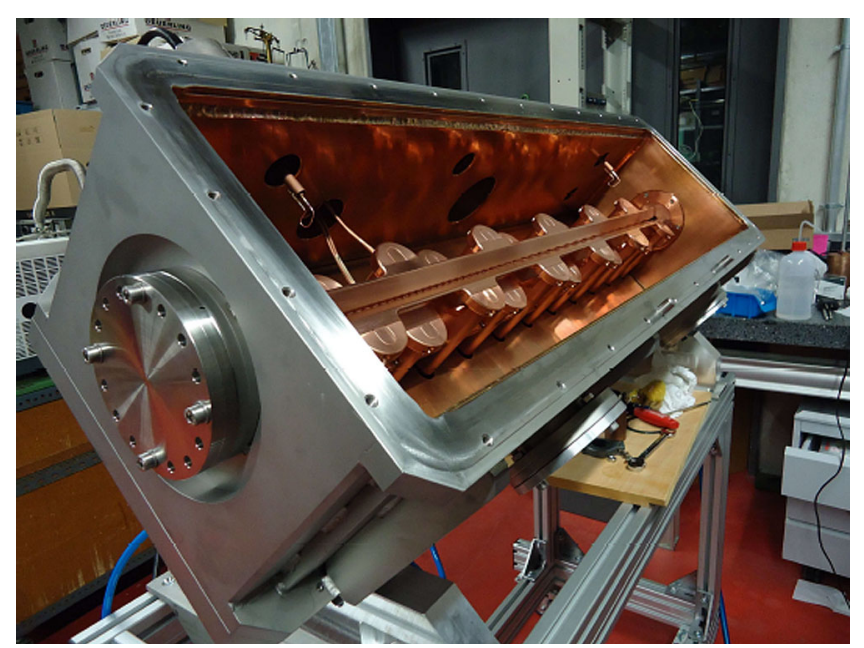

FIG. 4. The four-rod RFQ for FNAL during rf setup at IAP Frankfurt. This process includes adjustment of the resonant frequency and longitudinal voltage distribution, assembling of the piston tuner, matching of the coupling loop, as well as mechanical cross checks.

Based on these parameters, the four-rod RFQ was manufactured by Kress $\mathrm{GmbH}$ [7]. The rf setup was done at IAP Frankfurt, where the resonant frequency was adjusted to $201.6 \mathrm{MHz}$. The deviation in its longitudinal voltage distribution was set to less than $\pm 3 \%$. A more detailed description about the rf tuning procedure of the FNAL RFQ can be found in [8]. A picture of the actual structure is shown in Fig. 4.

\section{FIRST BEAM MEASUREMENTS}

\section{A. The test stand}

For the purpose of qualifying the RFQ for operations, a test stand was built that consisted of a $\mathrm{H}^{-}$source, LEBT, 


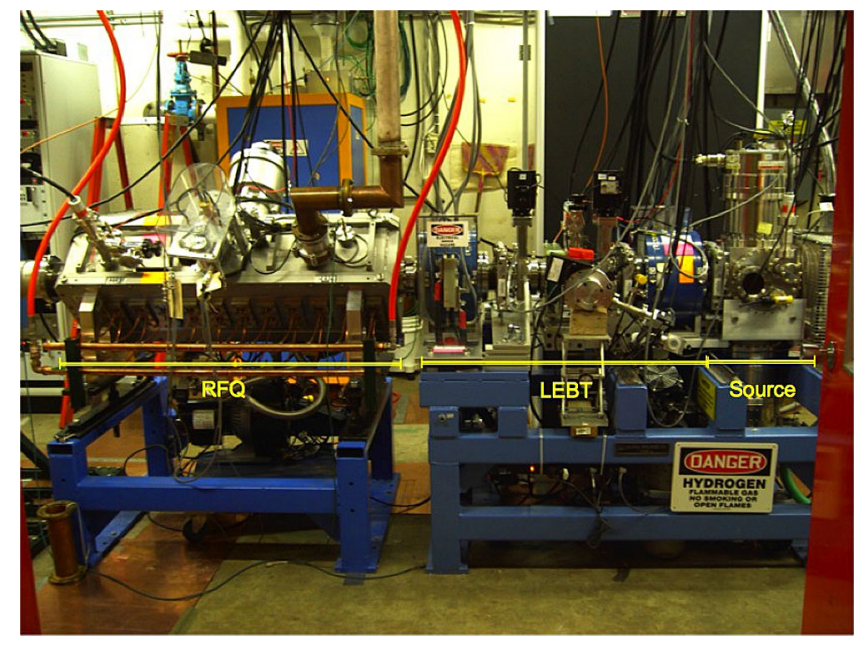

FIG. 5. The test stand consists of one $\mathrm{H}^{-}$magnetron source, the LEBT, and the RFQ. Instruments like emittance probes can be connected to the end of the RFQ.

and RFQ so that instruments could be connected to the end of the RFQ. See Fig. 5. The legitimacy of the results were also enhanced because the same $\mathrm{H}^{-}$source and LEBT components used here would be in the final installation.

The qualification tests for the RFQ are as follows: (i) Beam capture and bunching efficiency as a function of input power [9]; (ii) Output transverse emittance; (iii) Output beam energy.

For the transverse emittances, the measured C-W emittances are $1 \pi \mathrm{mm} \mathrm{mrad}$ rms normalized in both planes at $46 \mathrm{~mA}$.

Therefore, if the beam parameters are within these measurements, the new injector will work properly.

\section{B. Transmission efficiency and bunching as a function of RFQ power}

Both transmission and bunching are strong functions of the input power into the RFQ and will be discussed together because the following experiments show that they are intimately tied together. The experimental setup is shown in Fig. 6. For the transmission efficiency experiments, the Faraday cup is removed and the toroid is connected directly to the end of the RFQ. The RFQ transmission efficiency is defined to be the beam current measured at the exit of the RFQ with respect to the beam current measured at the LEBT toroid.

The results of the experiment are shown in Fig. 7. It is clear from this graph that maximum transmission occurs above $180 \mathrm{~kW}$ of RFQ power which is a factor of $\sim 2$ larger than the power specification in Table I. Furthermore, the transmission efficiency seen here is less than $58 \%$ which is a lot lower than $95 \%$ in the specifications. Also, it is not possible to operate the RFQ above $200 \mathrm{~kW}$ because the lifetime of the amplifier's tubes will be greatly lowered. In fact, it will be seen from the bunching experiment that the

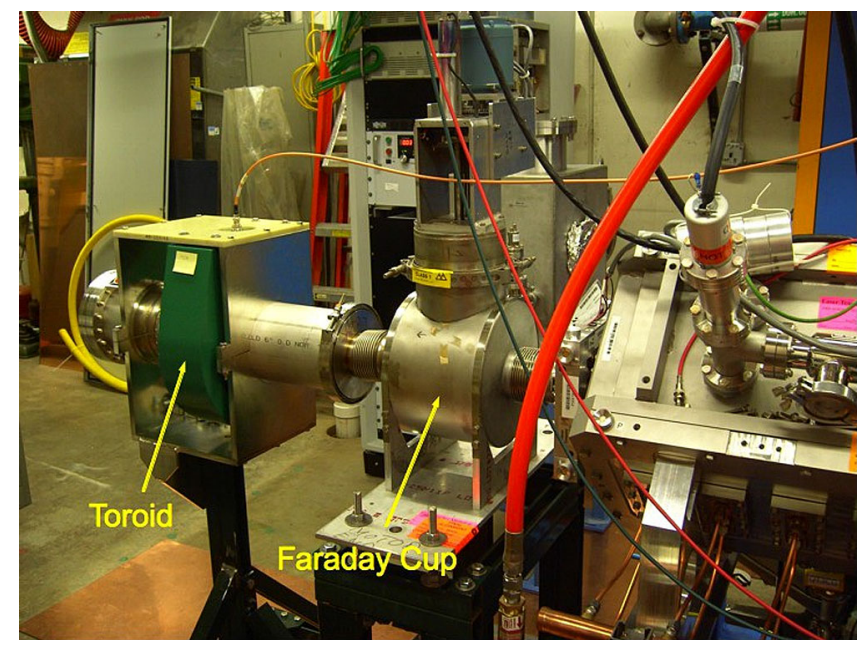

FIG. 6. A high bandwidth Faraday cup is connected to the end of the RFQ for bunch length measurements. For beam current measurements, the Faraday cup is removed and the toroid is connected directly to the end of the RFQ.

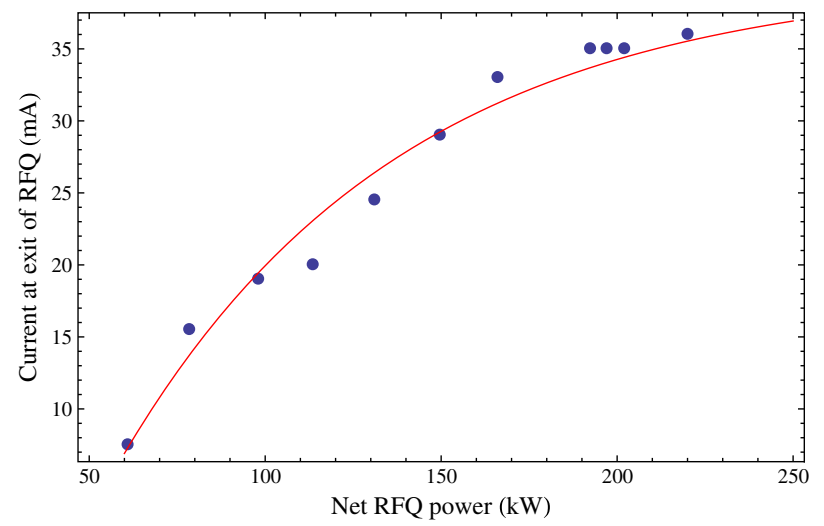

FIG. 7. The beam current (blue circle) measured with an oscillocope connected to a toroid installed at the end of the RFQ as a function of input power. The input current is $60 \mathrm{~mA}$ and the maximum transmission occurs above $200 \mathrm{~kW}$.

reason for the high power requirements is that bunching does not occur below $130 \mathrm{~kW}$.

For the bunching experiments, a fast Faraday cup [10] is connected downstream of the RFQ. The fast Faraday cup has a bandwidth of $15-20 \mathrm{GHz}$ and the signal is connected to a HP oscilloscope with a bandwidth of $6 \mathrm{GHz}$. Bunching does not occur below $130 \mathrm{~kW}$ and good bunching occurs above $180 \mathrm{~kW}$. Figure 8 shows the measured bunch signal on the fast Faraday cup.

Therefore, it is clear from these two sets of experiments that the power specifications have not been met.

\section{Output energy measurements}

The output energy of the RFQ is critical because of the small energy acceptance of $\pm 15 \mathrm{keV}$ of the first DTL. 

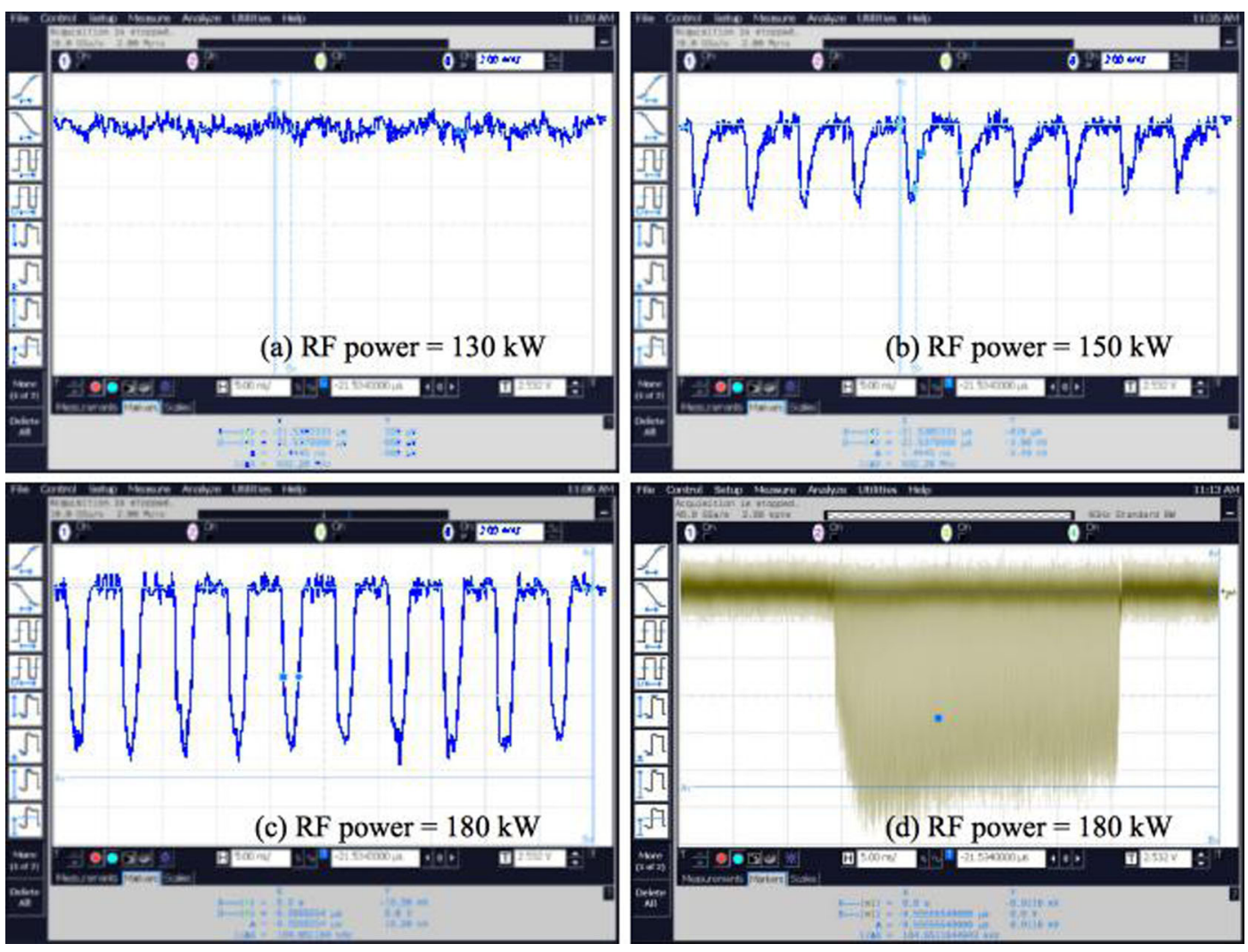

FIG. 8. Bunching as a function of RFQ power measured with a fast Faraday cup. Bunching starts above $130 \mathrm{~kW}$. There is no bunching at $130 \mathrm{~kW}$. (d) is the entire beam pulse seen on the Faraday cup at $180 \mathrm{~kW}$.

In the original plan, only the time-of-flight method (TOF) method would be used for the energy measurement. However, due to the energy error found by the TOF method, it was decided that an energy spectrometer (ES) would be built to confirm the TOF results.

\section{Time-of-flight method}

The TOF setup is shown in Fig. 9. Three button-type beam position monitors (BPMs) are installed on a $2.5^{\prime \prime}$ beampipe. It is essential to note that (i) Buttons are capacitive pickups and so it is the time differential of the wall current that is seen on an oscillocope; (ii) Bunches fill consecutive rf buckets during the rf pulse; (iii) The signal from each button is a multiple of the rf, $201.25 \mathrm{MHz}$.

Therefore, the fundamental idea behind the TOF method is that by measuring the phase difference $\Delta \varphi_{m n}$ between signals from the BPMs $m$ and $n$ (the numbering of the BPMs are shown in Fig. 9), the time $\Delta t_{m n}$ for the beam to go between them can be calculated using the formula

$$
\begin{aligned}
\Delta t_{m n} & =\frac{\left(N_{b} \times 2 \pi\right)+\Delta \varphi_{m n}}{2 \pi f_{\mathrm{rf}}}, \quad \text { where } \\
\Delta \varphi_{m n} & =\varphi_{n}-\varphi_{m} \text { for } n>m,
\end{aligned}
$$

where $N_{b}$ are the number of bunches between the two buttons and $f_{\mathrm{rf}}$ is the rf. However, notice that, if there are only two buttons, $N_{b}$ cannot be determined uniquely, and thus the phase term in the numerator is ambiguous by integer multiples of $2 \pi$. This predicament is solved by spacing three BPMs unequally longitudinally on the beampipe and measuring the phase difference between all possible BPM combinations. It is easy to show that only one $N_{b}$ can make the calculated $\Delta t_{m n}$ line up as shown in Fig. 10. From here the energy of the beam is easily calculated. An example of this method is shown in Fig. 10. Unexpectedly, the energy of the beam is found to be $(700 \pm 17) \mathrm{keV}$ rather than the required $750 \mathrm{keV}$.

Of course, this unexpected result caused a lot of consternation among the parties because if the RFQ modulations were correctly manufactured, the argument was that the beam energy cannot be incorrect. The usual reason is that the synchronous energy $E_{s}$ in cell $j$ is determined exclusively by the cell length $\ell$ and the $\mathrm{rf}$ wavelength $\lambda_{\mathrm{rf}}$,

$$
\beta_{s}(j)=2 \ell_{j} / \lambda_{\mathrm{rf}},
$$

where $\beta_{s}(j)$ is the relative velocity of the synchronous particle with respect to speed of light $c$ in the $j$ th cell that has length $\ell_{j}$. Notice that this formula is independent of the radial distance between rods. But in a gedanken experiment, one can imagine that if the rods are held infinitely far apart radially, then there can be no acceleration of the beam 


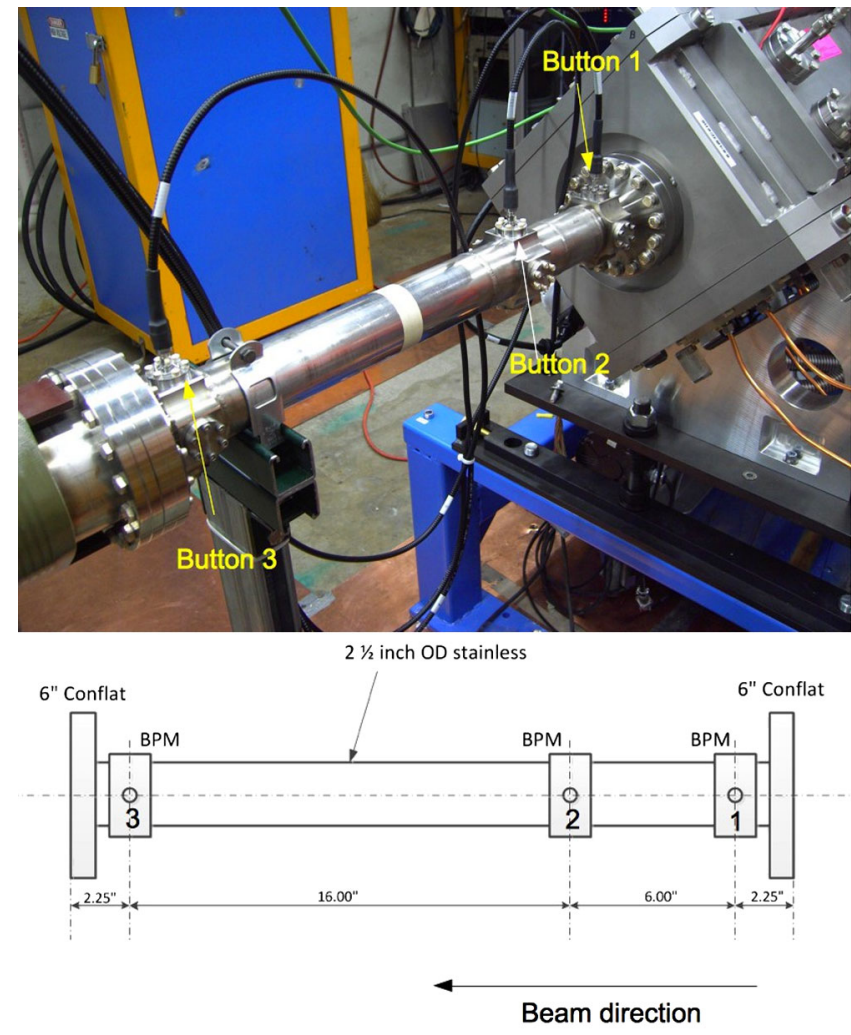

FIG. 9. The time-of-flight measurement setup consists of three button BPMs spaced unequally longitudinally on a $2.5^{\prime \prime}$ beam pipe. Notice the numbering on the BPMs.

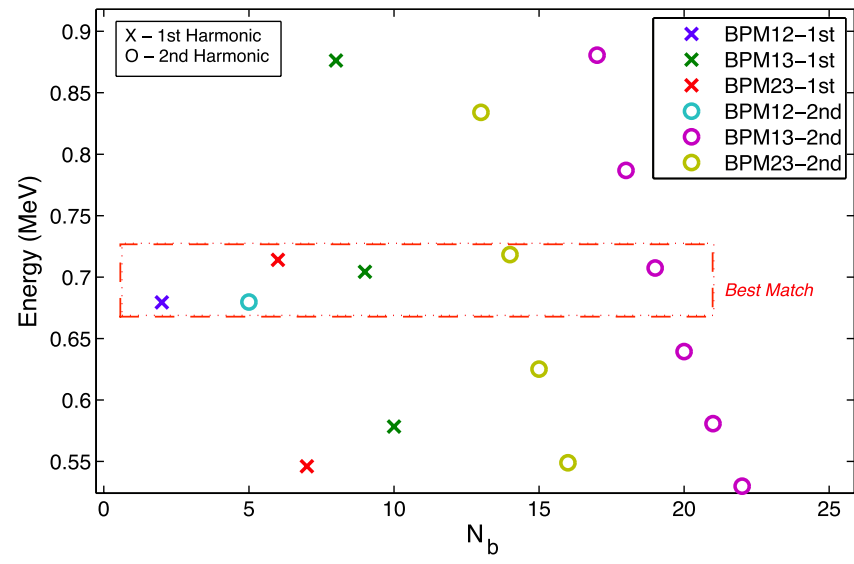

FIG. 10. By calculating the energy for different $N_{b}$ 's for all the button BPM combinations, the energy of the beam is found by finding the energy value where the points line up. In this example, both the fundamental (first harmonic) and the second harmonics are used in the calculation.

because the longitudinal accelerating electric field is zero, i.e., the output energy of the beam is equal to the input energy. However, the RFQ synchronous energy remains the same. Therefore, it is only when bunching is good that $E_{s}$ is the same as the mean energy $\langle E\rangle$. And in all the energy

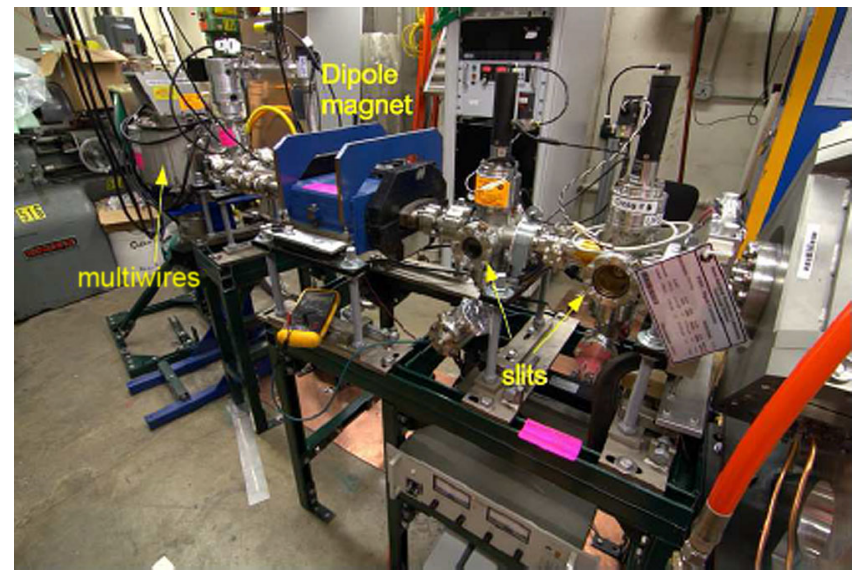

FIG. 11. A view of the ES setup for the energy measurement. The two slits downstream of the RFQ define the longitudinal axis. The deflected beam is measured on the multiwires which is $981.8 \mathrm{~mm}$ downstream from the center of the magnet.

measurements discussed in this paper, it is $\langle E\rangle$ that is measured and not $E_{s}$. This is exactly the computer simulation results that will be discussed in Sec. IVA 1 , where the rods are perturbatively distorted radially, $E_{s}=\langle E\rangle$ still holds true because bunching is still good.

\section{Energy spectrometer method}

The requirements for a successful spectrometer energy measurement (ES method) are manifold. They include (i) A dipole magnet with a very well measured magnetic field $B$ distribution. It needs to be calibrated for the integral of $B$ over the particle's path $l$ in the magnet $\int B d \ell$ versus input current $I$. In this case, $\int B d \ell$ has been measured to be better than $1 \%$. (ii) A double slit system to ensure that the direction of the beam can be accurately measured. (iii) A multiwire beam profile monitor whose position in the spectrometer line is accurately surveyed. (iv) An accurate survey of every component in the spectrometer. In this case, each component has been surveyed to \pm 1 mils $(= \pm 0.0254 \mathrm{~mm}$ ).

The setup is shown in Fig. 11 and for the interested reader, the details of the measurement can be found in [11].

The relationship between $\int B d \ell$ and the deflection angle $\theta_{d}$ of the beam is given by

$$
\theta_{d}=\frac{\int B d \ell}{B \rho}
$$

where $B \rho$ is the magnetic rigidity, and for negatively charged beam $B \rho=-p / c, p$ is the momentum of $\mathrm{H}^{-}$in units of $\mathrm{eV} / \mathrm{c}$, and $c$ is the speed of light in $\mathrm{m} / \mathrm{s}$.

Therefore, by changing the dipole current $I$ and measuring the deflection angle $\theta_{d}, B \rho$ can be obtained from the slope of the $\int B d \ell$ versus $\theta_{d}$ plot, and thus the energy of the beam can be found. 


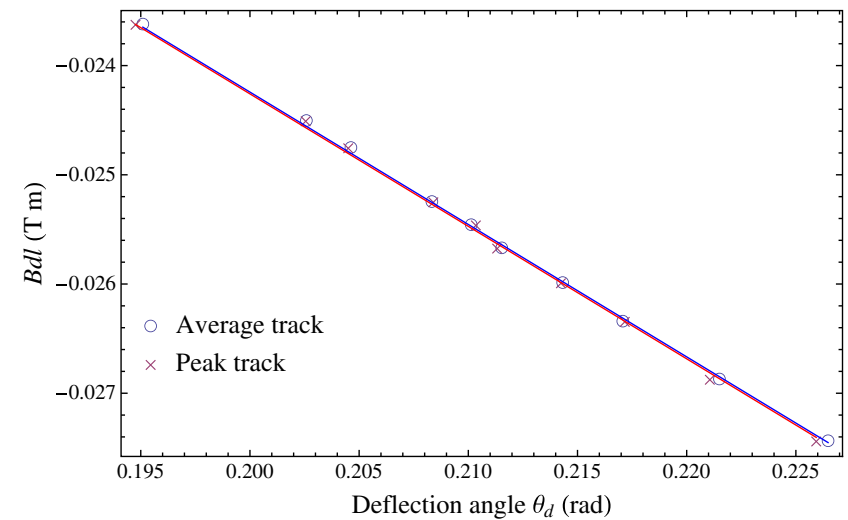

FIG. 12. The plots here show the size of $\int B d \ell$ required for the given deflection angle $\theta_{d}$. The circled points are the data when the peak of the angular distribution shown in Fig. 13 is tracked, while crossed symbol points are the data when the mean is tracked. The slope of the plot yields $B \rho$. In this case, tracking either the peak or the mean of the distribution gives the same slope.
An example of the application of this method is shown in Fig. 12 where the mean of the angular distribution shown in Fig. 13 is tracked as a function of dipole current at $156 \mathrm{~kW}$ of net RFQ power. The fitted slope $\langle B \rho\rangle$ is

$$
\langle B \rho\rangle=(0.12126 \pm 0.00005) \mathrm{T} \cdot \mathrm{m} / \mathrm{rad}
$$

From here, the mean energy of the beam $\langle E\rangle$ at $156 \mathrm{~kW}$ is easily calculated and is $\langle E\rangle=(703.3 \pm 0.6 \pm 5) \mathrm{keV}$ where the first error is statistical and the second is systematic.

Thus, both the TOF and ES method yield essentially the same result: the output energy of the RFQ is too low by more than $45 \mathrm{keV}$ or $6 \%$ of $750 \mathrm{keV}$ depending on the input power. This is far outside the required tolerance of $\pm 15 \mathrm{keV}$ or $2 \%$ of $750 \mathrm{keV}$.
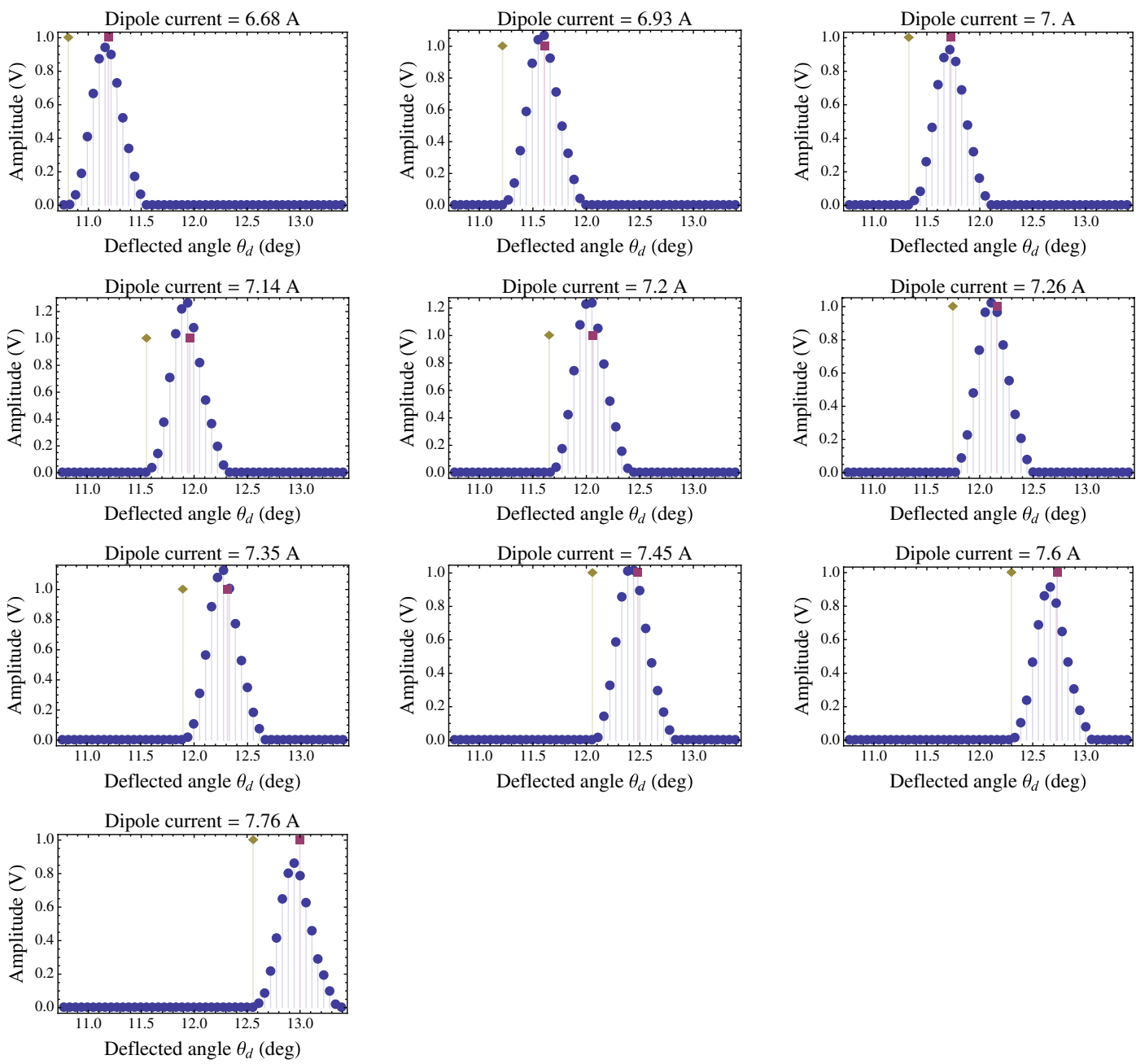

FIG. 13. The spectrometer data for the case when the RFQ is at $156 \mathrm{~kW}$ net power. As expected, the entire angular distribution moves when the dipole current is changed. The gray diamond indicates the expected deflection angle for $750 \mathrm{keV}$ beam and the red square indicates the expected deflection angle for $700 \mathrm{keV}$ beam. The peak of the distribution is clearly above $700 \mathrm{keV}$ and the mean energy is found to be $703 \mathrm{keV}$. 


\section{STUDIES ON RFQ OUTPUT ENERGY}

\section{A. Mechanical positioning of the electrodes}

As a first step in finding the source of the energy deviation, the electrodes of the RFQ were measured geometrically in situ. This measurement showed that the electrodes had shifted during transport. Their positions were displaced radially by up to one millimeter compared to what was measured in Frankfurt.

\section{Parmteq simulations $_{M}$}

In addition, simulations with Parmteq $_{M}$ have been done to check the influence of the misalignment of the electrodes. It is not possible to manipulate the values of the aperture $a$ manually in the Parmteq ${ }_{M}$ input file. To approximate the effect of radially bent electrodes in the simulations, the focusing strength $B$ was reduced locally compared to the original design, equivalent to enlarging the aperture by $1 \mathrm{~mm}$. This is based on the focusing strength of the RFQ as it is described for example in [12]

$$
B=\chi \frac{q e V}{m c^{2}} \frac{\lambda^{2}}{a^{2}},
$$

where $\chi$ is the dimensionless geometric parameter, $q$ is the charge state of the accelerated ion, $e$ is the elementary electric charge, $V$ is the electrode voltage, $c$ is the speed of light, and $\lambda$ is the rf wavelength. All other parameters of the RFQ design were kept constant for this study. Two different cases with deformed electrodes have been studied, one with a bigger aperture in the midsection of the structure (case 1 - red circle) and the other at both ends of the electrodes (case 2 - green upper triangle). The original design (black square) has a length of $1.2 \mathrm{~m}$ to reach the nominal output energy of $753 \mathrm{keV}$. Figure 14 shows the original aperture profile along the length of the RFQ compared to the changed parts of the aperture for both cases.

The results of these simulations for the synchronous energy and the transmission of the RFQ are presented in Fig. 15. In both cases, the displaced electrodes show no significant influence on the output energy of the RFQ and reach the design value of $753 \mathrm{keV}$ in $1.2 \mathrm{~m}$. This is the expected behavior: as the beam travels through the electrodes, the particles have to fit into the $\beta \lambda$ profile of the modulation. This profile determines the energy of the beam and leads to an automatic adjustment of the synchronous phase.

The second parameter that was studied is the transmission of the RFQ with the two cases of radial misalignment of up to $1 \mathrm{~mm}$. Case 2, with the bent electrodes at both ends, shows a minor reduction in transmission from $99.7 \%$ in the original design to $99 \%$. In contrast to case 2 , the displacement of case 1 has a stronger influence on the RFQ's transmission efficiency. When the aperture is enlarged by $1 \mathrm{~mm}$ in the middle of the electrodes the transmission drops down to $77.7 \%$. This can be understood

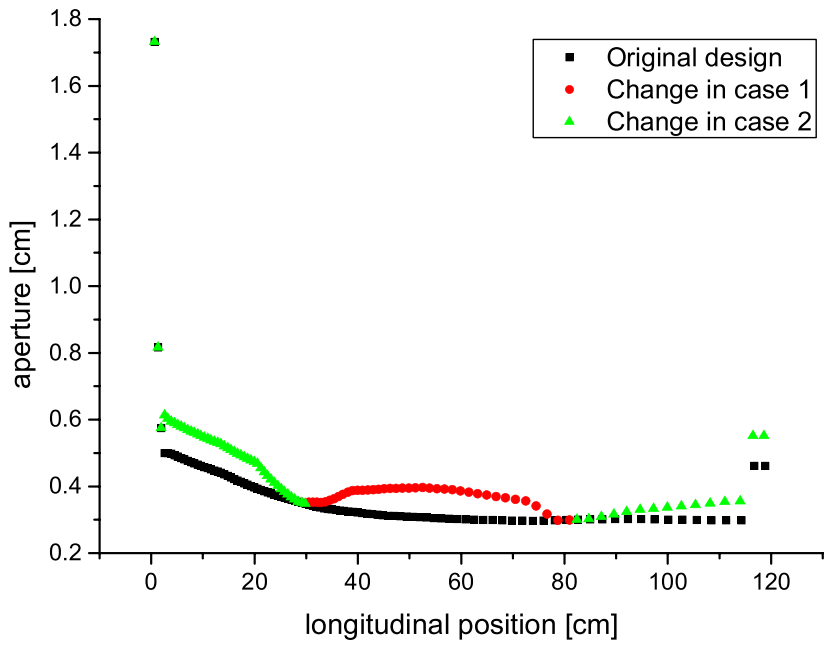

FIG. 14. Radial displacement of the electrodes with a $1 \mathrm{~mm}$ enlarged aperture in the middle section (case 1) or at both ends (case 2) of the electrodes.

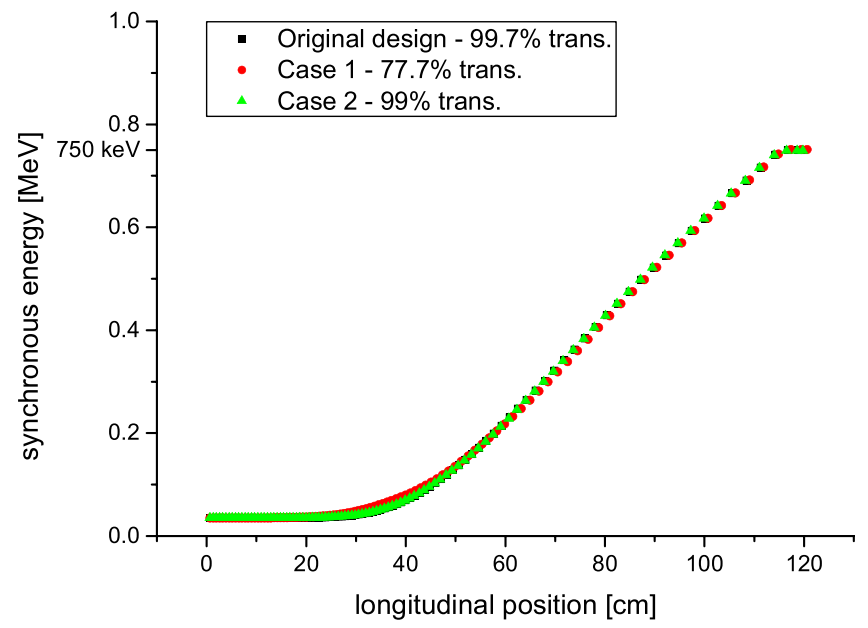

FIG. 15. Parmteq ${ }_{M}$ simulations: the radial displacement of the electrodes has no significant influence on the output energy of the RFQ. The displaced mid-section of the RFQ electrodes (case 1), where the bunching takes place, reduces the transmission from $99.7 \%$ to $77.7 \%$. While a displacement at both ends of the electrodes (case 2) leads to a minor reduction in transmission by $0.7 \%$.

by recalling from particle dynamics that the bunching of the beam takes place in this part of the RFQ. If the beam cannot be focused correctly in this section the bunching is reduced and particles are lost. As Fig. 16 shows, this effect can be compensated by applying a higher voltage on the electrodes so that the focusing strength can be increased. In this simulation a $20 \%$ higher voltage raises the transmission for case 1 back to $96 \%$.

\section{Mechanical measurements}

Since it has been shown that the radial alignment has an influence on the focusing properties of the quadrupole, the 


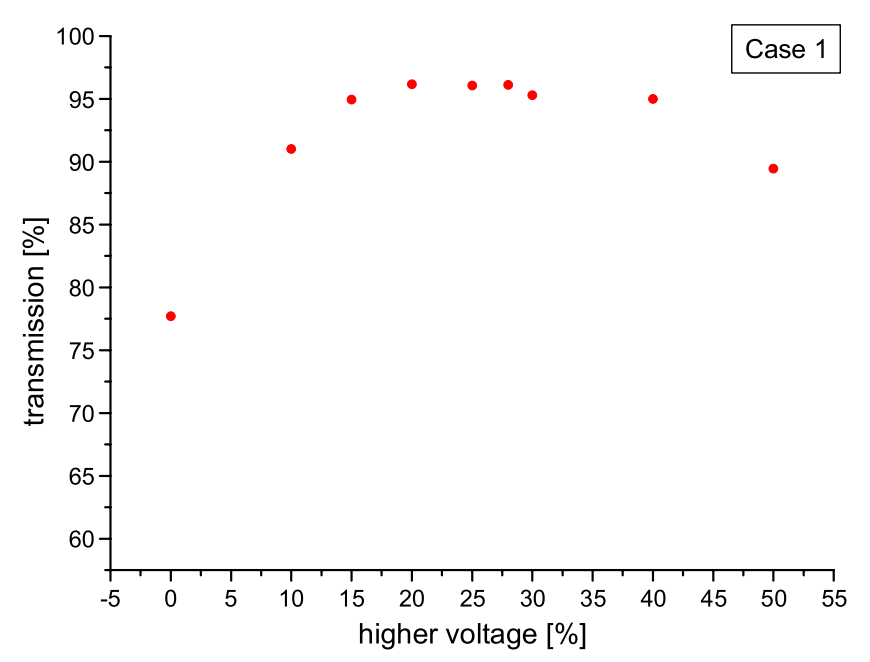

FIG. 16. Parmteq ${ }_{M}$ simulations: applying a higher voltage on the electrodes can compensate for the particle losses of the displaced electrodes of case 1 .

electrodes had to be readjusted. This required the dismantling of the electrodes. After the electrodes were removed, the electrode profiles in the longitudinal direction were measured to check whether the modulation agreed with the Parmteq $_{M}$ data and the machining data. The reason for this effort was to make sure that there were no manufacturing defects in the modulation that could have caused the energy error.

These measurements were executed by the Technical Division at FNAL using a CMM (coordinate measuring machine). The measurements have an accuracy of $0.05 \mathrm{~mm}$ and show that the modulation has no significant deviation between the geometric shape of the electrodes and the design data. In addition, the data translation from Parmteq $_{M}$ data to the machining data was verified. A comparison of the design data with the data used for machining showed absolutely no difference. This proves that the electrodes were manufactured using the right data.

Figure 17 shows the comparison between one electrode's design data for machining and the measurement data of the same electrode. This comparison does not show any significant deviation; that means the machining was within the manufacturing tolerance and the electrodes match the Parmteq $_{M}$ data.

In summary, the inspection of the longitudinal electrode design and especially verifying the cell length of the electrodes $\beta \lambda / 2$, leads to the conclusion that there are no errors in the design and manufacture of the electrodes. The insignificant $\beta \lambda / 2$ differences cannot explain the energy deviation of the RFQ. After these measurements, the electrodes were reassembled back onto the stems. This process was carried out with care in the radial and longitudinal positioning. The stem placement was checked before and after assembling the electrodes as well. After the correct placement of every single part in the tank, the

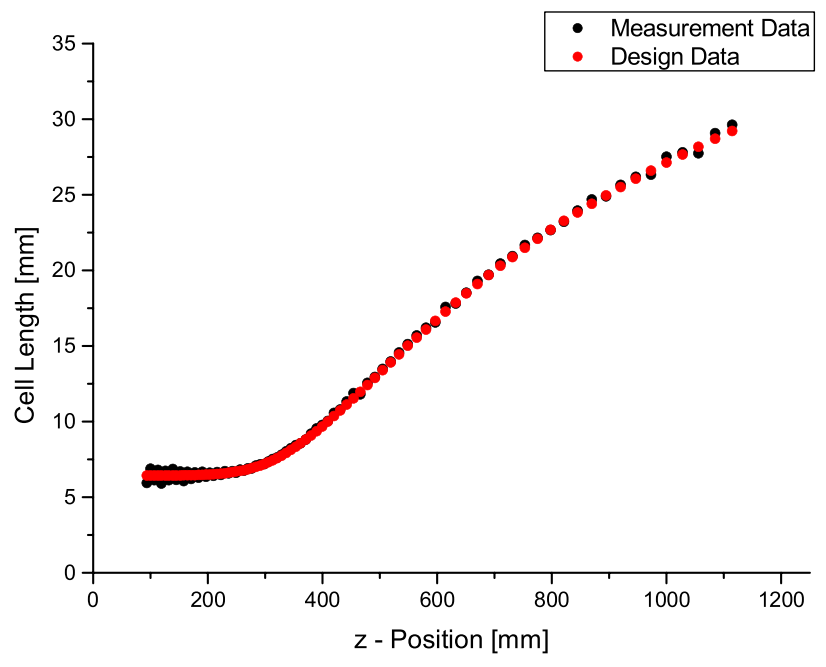

FIG. 17. Comparison of the machining data for the cell length (red) and its measurement (black). The differences between both are insignificant.

energy error remained. Nevertheless, it was an important step to exclude manufacturing and assembling errors as the reasons for the energy error.

\section{B. Electric field on beam axis}

To study effects that can lead to a change in the output energy of the RFQ, $E_{z}$ was scrutinized. This is the electric field component on the beam axis in the acceleration direction $z$. For an unmodulated quadrupole, there is no electric field on its symmetry axis. To produce an accelerating field component, a modulation profile, which was introduced by Kapchinsky and Teplyakov in [13], needs to be added. In order to determine the origin of the energy deviation, the design distribution of the accelerating field component $E_{z}$ can be compared with the results from rf simulations.

CST MWS calculation results for the longitudinal electric field component $E_{z}$ on axis, all scaled to the design voltage of $72 \mathrm{kV}$ between the electrodes, are presented in Fig. 18 for different models: A, B, and A without electrode modulation. These models are based on the imported engineering model of the four-rod FNAL RFQ but differ by the boundary conditions at the ends of the RFQ tank. In model A, the cylindrical beam pipes at the ends of the tank have both diameter and length of $100 \mathrm{~mm}$, while in model $\mathrm{B}$ they are shorter with $30 \mathrm{~mm}$ length and have a diameter of $15 \mathrm{~mm}$. In addition, the distribution of the tuning plates is slightly different in both models, see [14] for more details. The oscillating part of $E_{z}$ in Fig. 18 is due to the electrode modulation dictated by the beam-dynamics design. The curves for models A and B perfectly overlap in that part, while in model A without modulation the oscillations are absent, as expected. However, in addition to the oscillating pattern, there are noticeable peaks of the longitudinal field near the entrance and the exit of the 


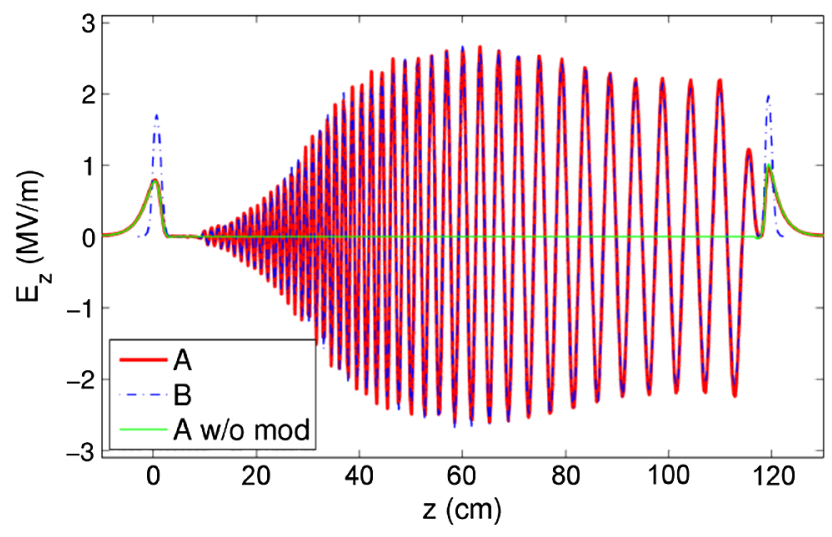

FIG. 18. Calculated longitudinal component of electric field on axis $E_{z}$ in RFQ MWS models A, B, and A without modulation.

structure, mainly located in the gaps between the tank wall and the electrodes. They are exactly the same in model A with and without modulation, but even stronger in model B, where the beam pipes are narrow. The end-gap longitudinal field exists because the quadrupole symmetry is broken near the RFQ ends; it vanishes in a perfectly symmetric structure like a four-vane RFQ. One can also show that 3D electrostatic computations in model A with the vanes charged as a quadrupole reproduce the oscillating pattern of $E_{z}$ but not the near-end peaks [14], which confirms their rf origin. Such nonzero potential studies on the beam axis in RFQs have been presented in [15] for a RFQ with one pair of electrodes at ground potential and [16] for a four-rod type with symmetric supports.

In order to match the output beam ellipse to the downstream Linac, the RFQ was designed with a Crandall cell at the end of the electrodes. It consists of two parts: a transition cell [17] to merge the modulated acceleration cell to a symmetric point, with the same aperture for $x$ and $y$ electrodes, and an unmodulated end part. This unmodulated section focuses the beam on a fitted length to form a symmetric transverse output distribution of the bunch.

In the FNAL RFQ design with a typical Crandall cell, the synchronous particle enters the gap behind the electrodes at a phase of $65^{\circ}$ in the sinus convention. This is in contrast to a conventional RFQ design that ends directly with an acceleration cell, where the synchronous phase is $60^{\circ}$ in the middle and $150^{\circ}$ at the exit of the cell [18]. This $90^{\circ}$ difference in the synchronous phase between a Crandall and a conventional end cell at the end of the electrodes means that $E_{z}$ is on its rising edge at about $90 \%$ of its maximum amplitude in the case of a Crandall cell, while in the case of a conventional end cell it is at about $50 \%$ on the falling edge.

Since the energy gain in the gap is dependent on the transit time factor and the phase [17], the field peak in the gap between the electrodes and the tank wall has a strong influence on the output energy. It is the combination of a Crandall cell and the gap field between the electrodes and the tank wall that leads to the measured energy deviation. The source of this gap field is discussed below.

\section{Tuning plates}

The results of the rf setup have been presented in [8]. The tuning plates are at relatively high positions between the stems and to gain more flexibility in tuning, copper half cylinders were added to some rf cells. The influence of different mechanical elements of the four-rod RFQ has been studied in [19].

One has to keep in mind, that the resonant structure of the four-rod RFQ at $200 \mathrm{MHz}$ is considerably smaller than at $100 \mathrm{MHz}$. This makes the FNAL RFQ altogether more sensitive to external influences on its field geometry [20] than $100 \mathrm{MHz}$ RFQs.

Figure 19 shows $E_{z}$ at the entrance of the RFQ, where the electrodes start at $z=25 \mathrm{~mm}$. Two tuning options are compared: one with tuning plates in the highest position possible in the rf cells (red) and the other with the tuning plates (black) at the lowest position. In this simulation without modulation on the electrodes, $E_{z}$ should be zero along the entire length of the RFQ with minimal fluctuations where the stems are located. But outside the electrodes, a field component exists at the entrance and the exit of the electrodes. This bump in the electric field $E_{z}$ can be reduced by a factor of 1.2 by lowering the tuning plates.

\section{Opening at the end of the tank}

The influence of the high tuning plates on the boundary fields is significant, but not strong enough to cause the irregularities in $E_{z}$ shown in Fig. 18. So more detailed studies on the boundary conditions formed by the tank design have been done. An important element here is the insert at the wall of the tank which is used to shield

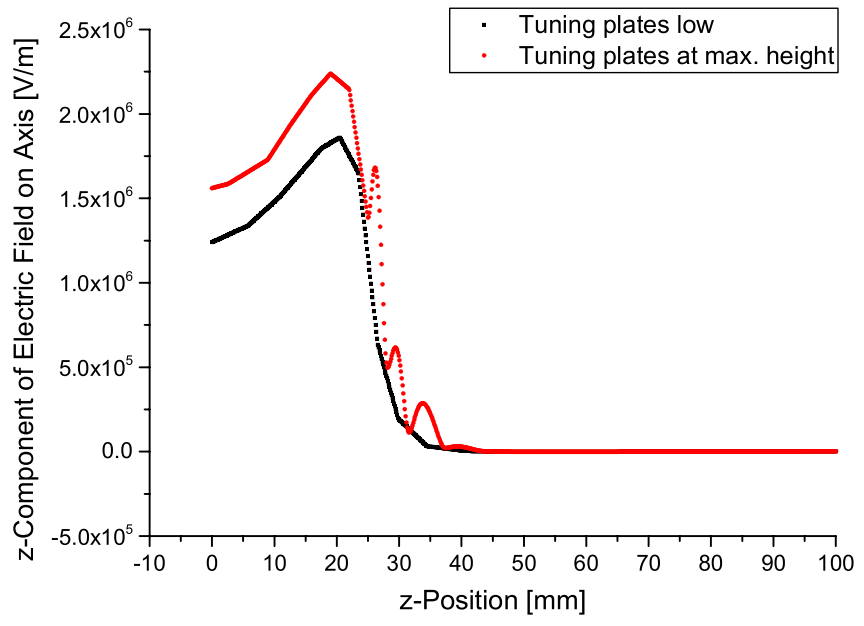

FIG. 19. $z$-component of electric field on axis at the entrance of the RFQ with variation of the tuning plate's height. The gap field increases by a factor of 1.2 when the tuning plate in the first cell is raised from its minimum to its maximum position. 


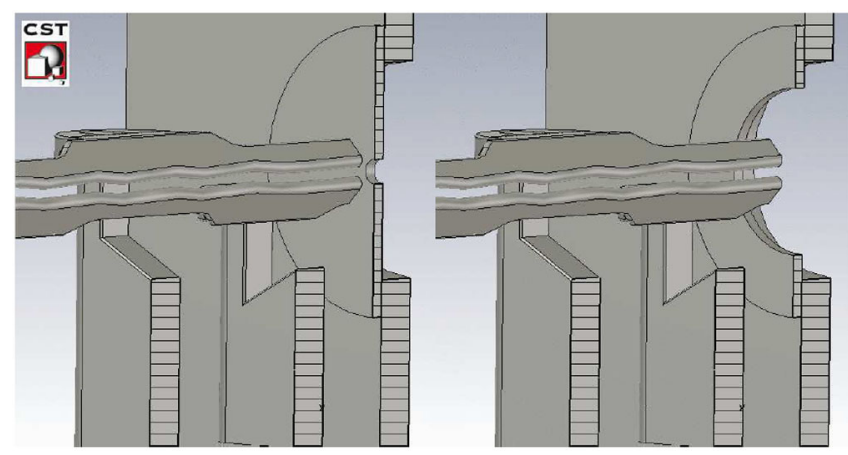

FIG. 20. Variation of the rf shielding insert's opening radius in the simulation model.

neighboring devices from rf leaking out of the RFQ. A detailed view with a narrow and a wide aperture of that part in the four-rod RFQ is given in Fig. 20. Figure 21 shows $E_{z}$ in the same $z$-range as Fig. 19 with different aperture radii in the shielding plate. Both fields are at 0 -phase, at the full amplitude of the electric field. In Fig. 21, the strong impact of the insert's aperture is clearly seen. In this case, opening the aperture radius from $3 \mathrm{~mm}$ to $75 \mathrm{~mm}$ suppresses the field peak between the electrodes and the tank by a factor of 5 . Changing it to $40 \mathrm{~mm}$ results in a factor of 4.5 lower field peak. But the main value for the beam dynamics is the integral of the field. This is shown in Fig. 22 where the integral of $E_{z}$ along the beam axis is compared. The $3 \mathrm{~mm}$ aperture radius of the rf shielding has been chosen to be the reference voltage and has been set to $100 \%$. In this comparison, the voltage on the axis is lowered by more than $15 \%$ with the $75 \mathrm{~mm}$ aperture. In [15] a similar case was studied as mentioned before. Also for this case it was shown that the aperture radius at the end of a RFQ has a strong influence on the axis potential.

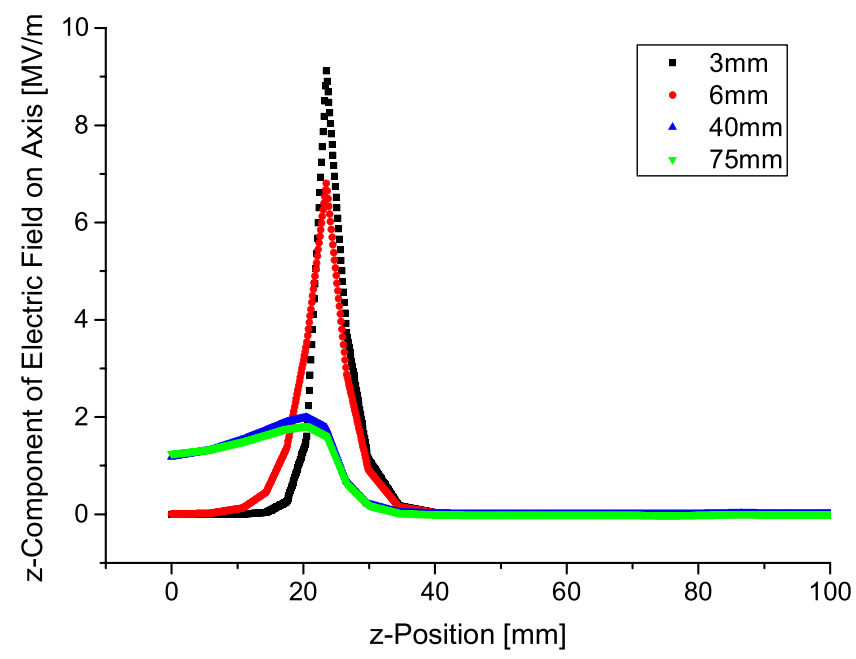

FIG. 21. $z$-component of electric field on axis with variation of the inlets opening radius. This variation has a strong influence on the gap field of the four-rod structure. By opening the aperture of the rf shielding, a reduction of the peak by a factor of 4.5 can be achieved.

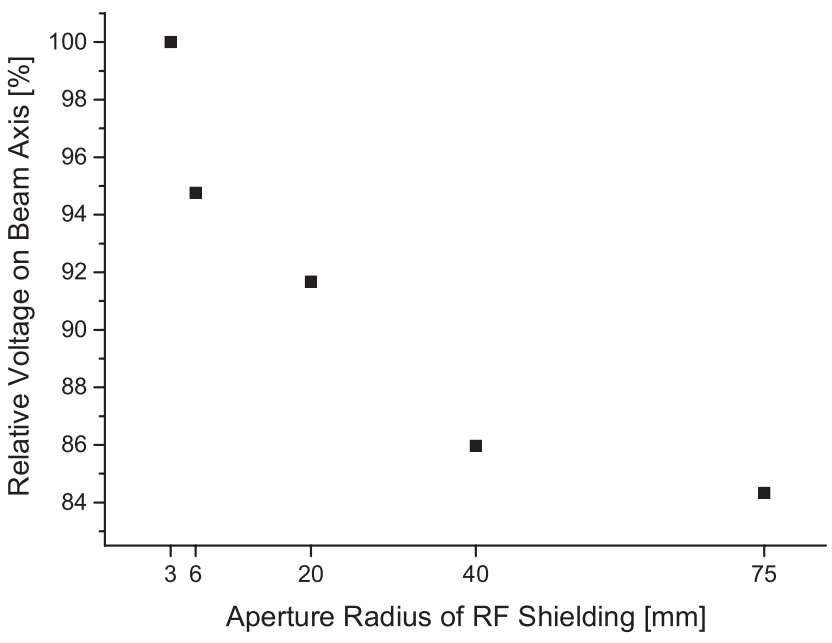

FIG. 22. Comparison of the relative voltage on axis when the aperture of the $\mathrm{rf}$ shielding is opened. The reference voltage is the value for the $3 \mathrm{~mm}$ aperture radius which has been set to $100 \%$.
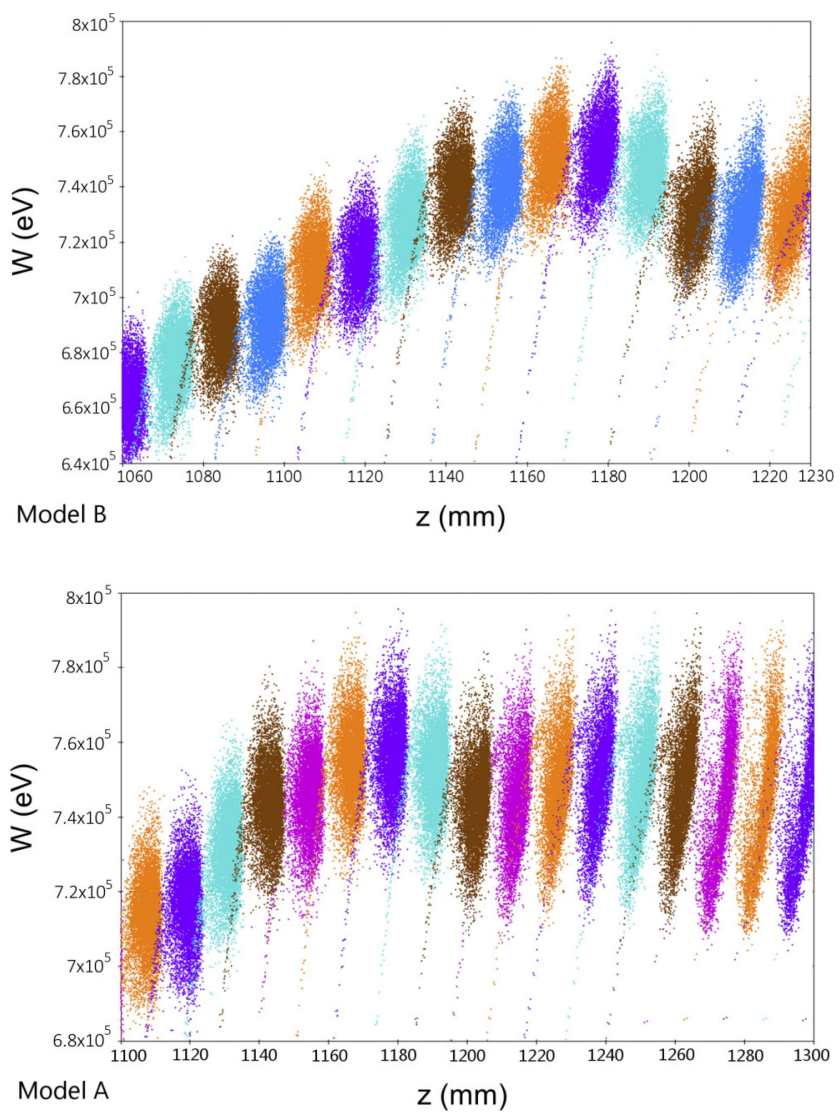

FIG. 23. CST particle studio simulation results for particle energies versus the bunch longitudinal position near the RFQ exit in model A (bottom) and B (top). In the case of a small tank opening (B) the particles are decelerated behind the electrodes, while they keep their energy with the wide opening (A). 
To confirm that the influence of the size of the aperture on the field peaks is strong enough to be responsible for the measured energy deviation, it was simulated using CST particle studio. Detailed particle-in-cell simulations reproduce the output energy decrease of about $20 \mathrm{keV}$ when the end-flange opening changes from wide to narrow. Figure 23 presents the results of the particle evolution in the final section of the RFQ. It shows five overlapped snapshots of the longitudinal phase space $z-W$ near the RFQ exit, each in its own color, separated in time by $1 \mathrm{~ns}$ (see [14] for details). Each bunch in Fig. 23 contains about 9000 macroparticles with some energy spread. The bunch energy (the average energy of the bunch particles) reaches the design energy of $750 \mathrm{keV}$ near the end of the electrodes. But while the bunches remain at the design energy with a wide opening (model A), in the case of a small aperture at the end of the RFQ (model B), the particles are decelerated in the gap between the electrodes and the tank wall by about $20 \mathrm{keV}$. This value is consistent with that measured in the experiment discussed in Sec. III C.

\section{ACHIEVEMENT OF REQUIREMENTS}

Following the findings of the previous section a modification to the RFQ was performed. There were two main challenges in this project: (i) to optimize the tuning and the opening at the end of the tank so that the boundary fields can be reduced and the output energy corrected; (ii) to reconstruct the four-rod geometry in order to lower the power requirements of the RFQ. The advantage of the fourrod structure is that it can be built in a modular way, because it is a current resonator with negligible currents on its vacuum tank walls. This fact offers the opportunity to modify or exchange parts of the RFQ easily.

The rf shielding plate at the end of the RFQ was removed completely as one can see in Fig. 24. The same shielding at the RFQ entrance was not removed because it led to problems with rf leaking into the Einzel lens in front of the RFQ. As a compromise its hole was enlarged from $20 \mathrm{~mm}$ to $31.75 \mathrm{~mm}$ diameter.

To lower the tuning plates, a higher resonant frequency of the RFQ needed to be achieved. This could be realized

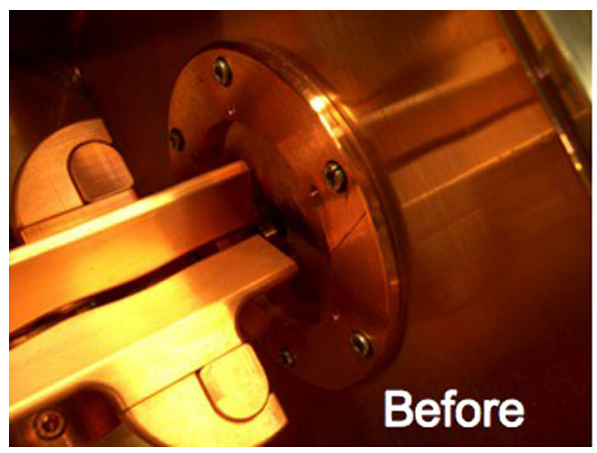

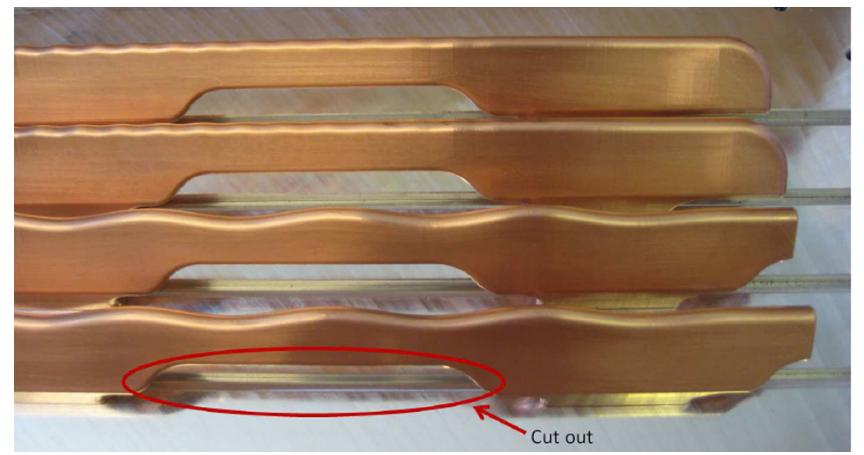

FIG. 25. Picture of the capacitance reduced new electrodes with cut outs on their backs.

following Thomson's law $f_{0}=1 / 2 \pi \sqrt{L C}$ by reducing the capacitance of the resonant structure. New electrodes have been built which are much thinner due to cut-outs between the connection points to the stems. The installation of even thinner electrodes in the four-rod RFQ is constrained by their mechanical stability. A picture of these new electrodes is presented in Fig. 25. Simulations of both types of electrodes predict a frequency change of plus $5 \mathrm{MHz}$ with respect to the thick electrodes. The higher frequency gives the opportunity to reduce the height of the tuning plates.

\section{A. Beam measurements after modifications to the RFQ}

\section{Output energy with end plate removed and thick electrodes}

The most important experiment is to measure the output energy after the end plate is removed (but still with the thick electrodes). The ES method discussed in Sec. III C 2 is used for the measurement. Like before, $\int B d \ell$ as a function of $\theta_{d}$ is plotted (shown in Fig. 26) and the slope measured to give

$$
\langle B \rho\rangle=(0.1257 \pm 0.0001) \mathrm{T} \cdot \mathrm{m} / \mathrm{rad} .
$$

From this value, the mean energy is found to be $(756 \pm 1 \pm 5) \mathrm{keV}$ at $167 \mathrm{~kW}$ of net RFQ power.

An example of the energy distribution measured on the multiwires can be seen in Fig. 27. And from this

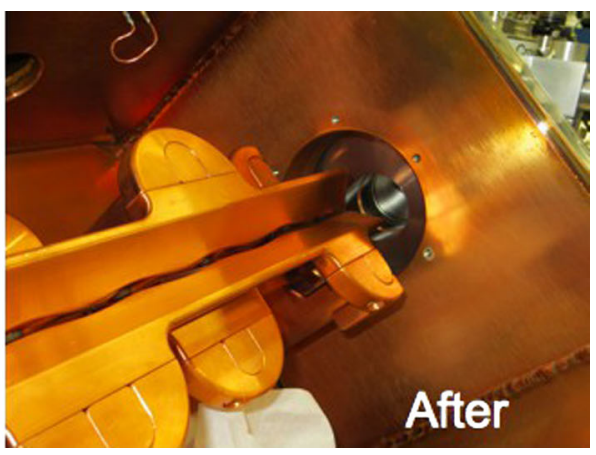

FIG. 24. The rf shielding was removed at the high energy end of the RFQ and its aperture was enlarged at the low energy side. 


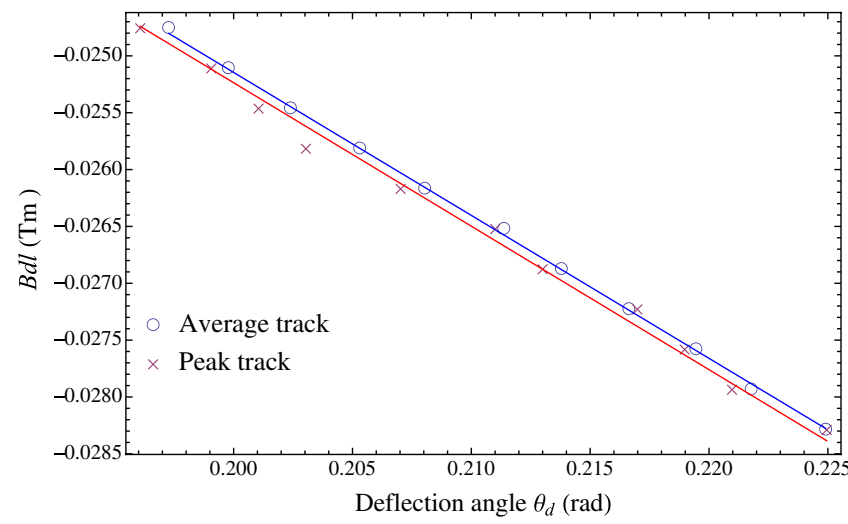

FIG. 26. Like in Fig. 12, the circles are the data points when the mean of the distribution is tracked and crossed symbols data points are when the peak of the distribution is tracked.

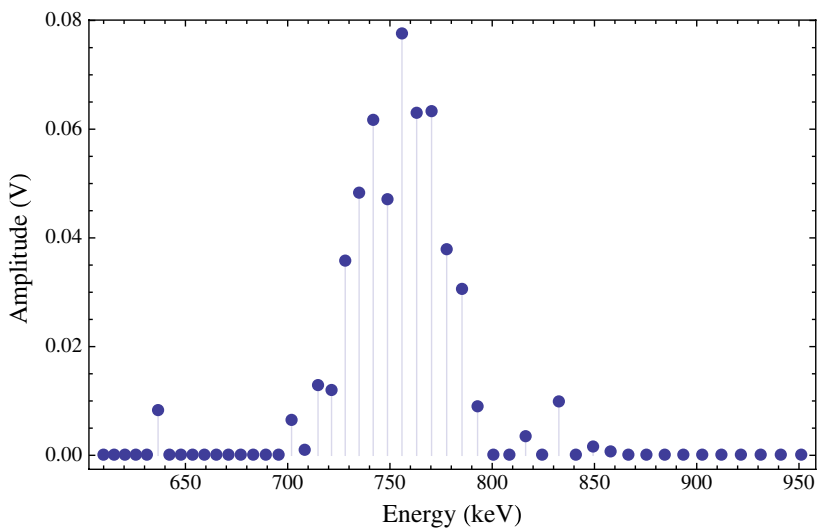

FIG. 27. Energy spectrum of the $\mathrm{H}^{-}$beam after the RFQ modifications. The energy of this spectrum is $(756.5 \pm 0.5) \mathrm{keV}$ with the dipole current at $7.5 \mathrm{~A}$.

distribution, the mean energy of the beam is $(756.5 \pm 0.5) \mathrm{keV}$.

Also measured is the output energy and the energy spread of the beam as a function of RFQ net power shown in Fig. 28. It is obvious that both of these parameters have a small energy dependence on the RFQ power. The average energy found here is $(758 \pm 1) \mathrm{keV}$ and the energy spread is $(15.4 \pm 0.4) \mathrm{keV}$ which is within the specified energy tolerance. The hypothesis for the energy dependence is that the $E_{z}$ field at the boundary still has some influence on the beam even with the rf shielding removed.

From these measurements, the energy problem is considered resolved because the measured energy is within $1 \%$ of $750 \mathrm{keV}$.

\section{Bunching and power requirements with thin electrodes}

When the thick electrodes were replaced with the thin electrodes their capacitance was reduced and with that the
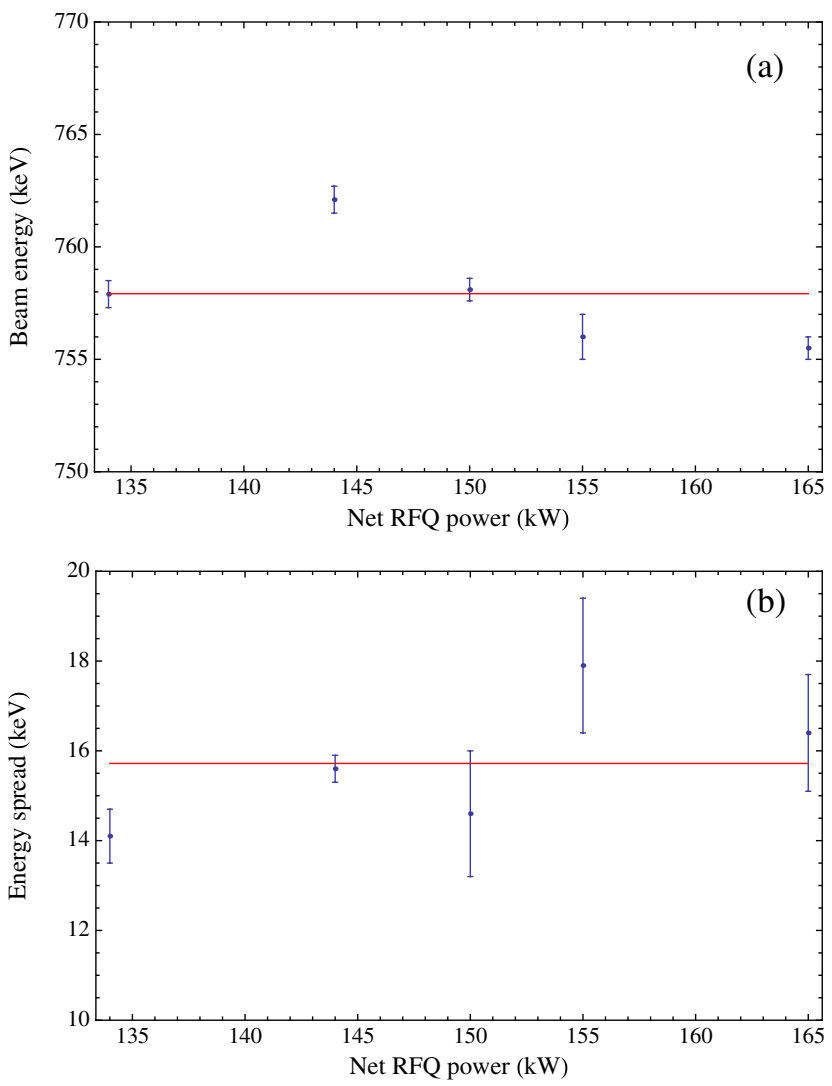

FIG. 28. (a) The mean output energy of the beam (shown here measured with the average tracking method) has a small energy dependence on the net RFQ power. The effect is $<1 \%$. The red line is drawn at $758 \mathrm{keV}$ which is the average energy of all the data points shown here. (b) The average energy spread of the beam is $(15.4 \pm 0.4) \mathrm{keV}$ (red line) in this net RFQ power range.

shunt impedance of the resonator was improved. The definition of the shunt impedance $R_{P 0}$ [21] that is used in order to define the average power $\bar{N}$ to achieve an electrode voltage amplitude $U_{0}$ is

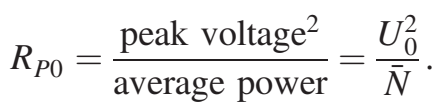

When this formula is applied with the measured values shown in Table III, the rf power ratio between thin and thick rods is found to be

$$
\frac{\mathrm{rf} \text { power for thin rods }}{\mathrm{rf} \text { power for thick rods }}=0.71 \text {. }
$$

TABLE III. Design parameters of the FNAL RFQ.

\begin{tabular}{lcc}
\hline \hline & Thick electrodes & Thin electrodes \\
\hline Shunt impedance $R_{P 0}$ & $31.7 \mathrm{k} \Omega$ & $44.6 \mathrm{k} \Omega$ \\
Power $\bar{N} @ U_{0}=72 \mathrm{kV}$ & $163.6 \mathrm{~kW}$ & $116.3 \mathrm{~kW}$ \\
\hline \hline
\end{tabular}



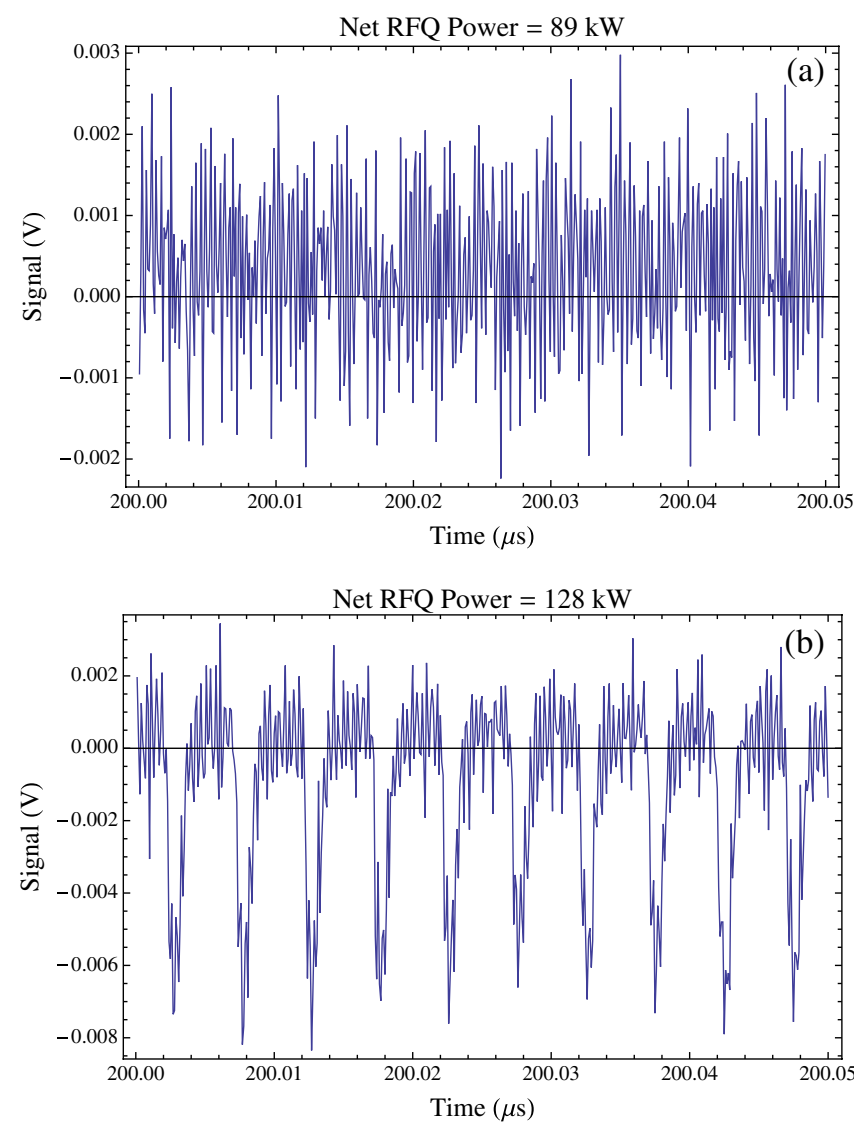

FIG. 29. The signal from the fast Faraday cup. (a) Below $100 \mathrm{~kW}$ of input rf power, there is clearly no bunching (b) at $128 \mathrm{~kW}$, the beam is bunched.

Therefore, from these calculations the expected power reduction required for bunching is about $29 \%$.

For the bunching experiment, the fast Faraday cup is connected to the end of the RFQ like in Fig. 6. Figure 29 shows examples of nonbunching and bunching for two different rf power levels. With the thin electrodes bunching

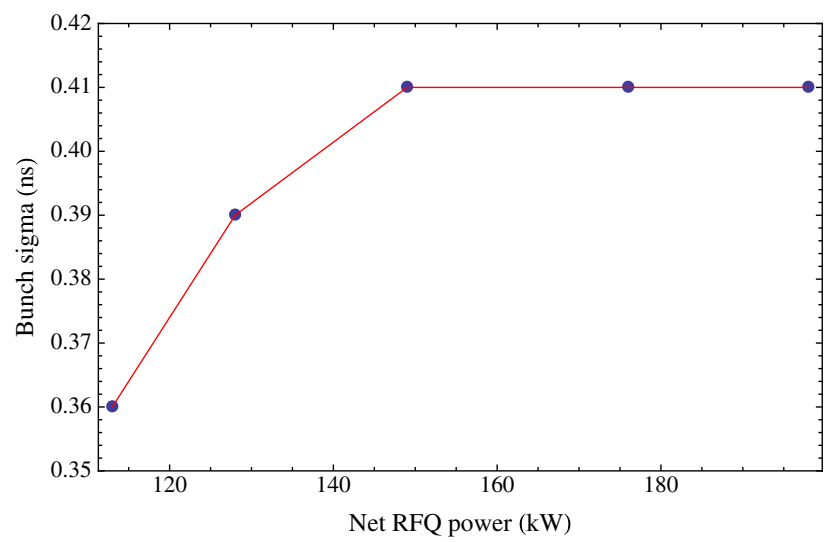

FIG. 30. This plot shows that the bunch length increases as power is increased until $150 \mathrm{~kW}$ where it reaches a stable length of $0.41 \mathrm{~ns}$ (sigma). occurs above $100 \mathrm{~kW}$. This can be contrasted to the thick electrodes bunching data shown in Fig. 8 which shows that bunching does not occur at $130 \mathrm{~kW}$. So by replacing the electrodes, the power required for bunching has been reduced by about $23 \%$ which is consistent with the expected values compared to the improved shunt impedance as it is discussed above. In fact, stable bunching occurs above $150 \mathrm{~kW}$ which can be seen in Fig. 30 .

\section{Output beam current with end plate removed and thin electrodes}

For operations, the minimum current required at the exit of the RFQ is $40 \mathrm{~mA}$ in order to do no harm. In the injector design, the $\mathrm{H}^{-}$beam is focused into the RFQ with a solenoid in front of the RFQ. Space charge forces tend to overcome the solenoidal focusing and so gas focusing of the beam plays an important role for maximizing the output current of the RFQ [22]. Figure 31 shows the effect on the beam current out of the RFQ as a function of LEBT gas pressure. From these experiments, the LEBT pressure of $>3 \times 10^{-6}$ Torr will be used to produce $44 \mathrm{~mA}$ beam for the DTL.

\section{Emittance measurements with end plate removed and thin electrodes}

The emittance measurements also show a dependence on rf power that can be seen in Fig. 32. The transverse emittance stabilizes to $\epsilon_{x}=0.6 \pi \mathrm{mmmrad}$ and $\epsilon_{y}=$ $0.5 \pi \mathrm{mm} \mathrm{mrad}(1 \times \mathrm{rms}$ normalized $)$ above $170 \mathrm{~kW}$. In this experiment, the input beam current into the RFQ is $65 \mathrm{~mA}$ while the output beam current is dependent on beam power. The transverse beam emittance is asymmetric because the beam coming out of the source is asymmetric. This can be traced to the dipole field in the magnetron source and can be easily proved by rotating the source by $90^{\circ}$, and in this case the emittances are exchanged, i.e., $\epsilon_{x}$ becomes smaller than $\epsilon_{y}$. The measured emittances can be

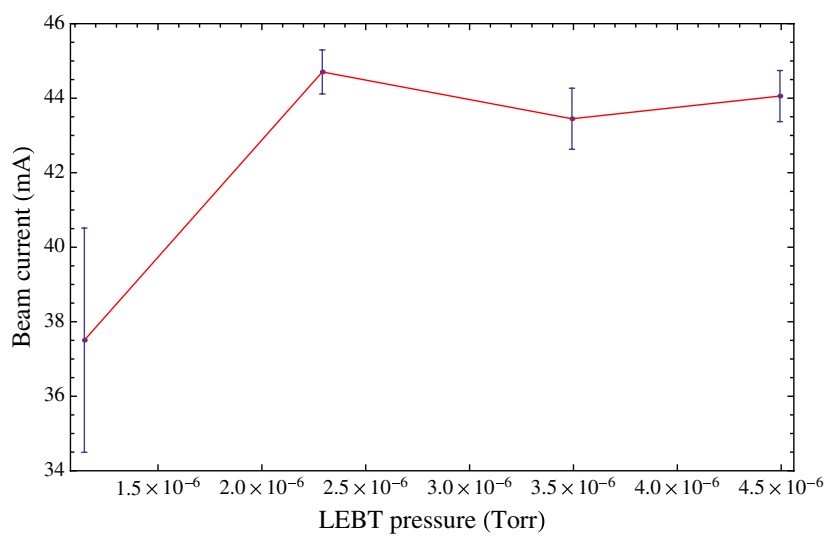

FIG. 31. The output current of the RFQ as a function of LEBT pressure. Under $2 \times 10^{-6}$ Torr the gas focusing is not strong enough to overcome space charge. 


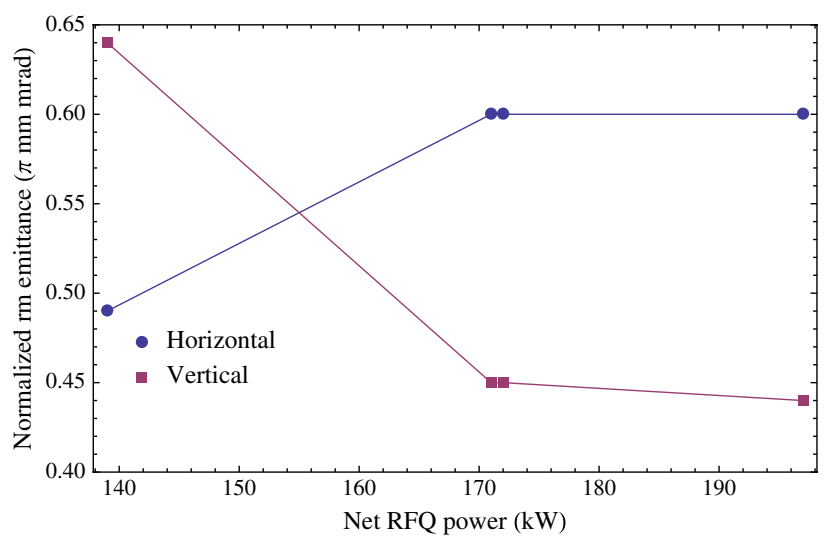

FIG. 32. The effect on emittance as a function of RFQ power. The emittances stabilize above $170 \mathrm{~kW}$.

compared to the specified emittance shown in Table I. They are clearly larger then specifications, however they are smaller than the measured emittance of the CockcroftWalton by $40 \%$ in the $x$-plane and $50 \%$ smaller in the $y$-plane and so these values are acceptable for operations.

\section{SUMMARY}

The RFQ injector has replaced the C-W's and the first beam that was used to treat patients in the neutron therapy facility at FNAL was delivered on 13 Dec 2012. However, the peak beam current at the start of the DTL was below $40 \mathrm{~mA}$ at that time. On 25 Jun 2013 after the addition of extra correctors at the exit of the RFQ, better energy matching, increased gas focusing, and better tuning, $40 \mathrm{~mA}$ of peak current at the beginning of the DTL was achieved. See Fig. 33. More work still has to be done to consistently deliver $40 \mathrm{~mA}$ to the start of the DTL as the NOVA program ramps up to accept $700 \mathrm{~kW}$ of beam power from the main injector. The current in the original plan was $>50 \mathrm{~mA}$ of current at the start of the DTL. So, in the interim, simulations with TRACK have been started in order to understand the reason why the transmission efficiency is only about $67 \%$ from the $\mathrm{H}^{-}$source to the start of the DTL and whether there are any other ways to increase transmission.

Stimulated by the challenges of this project and the points learned about the boundary fields in four-rod RFQs, more studies on this subject have followed. They reveal that the four-rod structure has intrinsic boundary fields in the gap between the electrodes and the tank wall, which can influence the output energy of the beam considerably. However, these fields can be handled properly in the rf design of a four-rod RFQ and there are various ways to minimize them to an acceptable level. A summary of simulations on the effect of the RFQ design on these fields and their impact together with more details about the simulation techniques are presented in [23] and [14]. These papers show that with a careful rf design coupled with modern simulation tools, the field distributions can be optimized to have a well-performing, reliable four-rod RFQ.

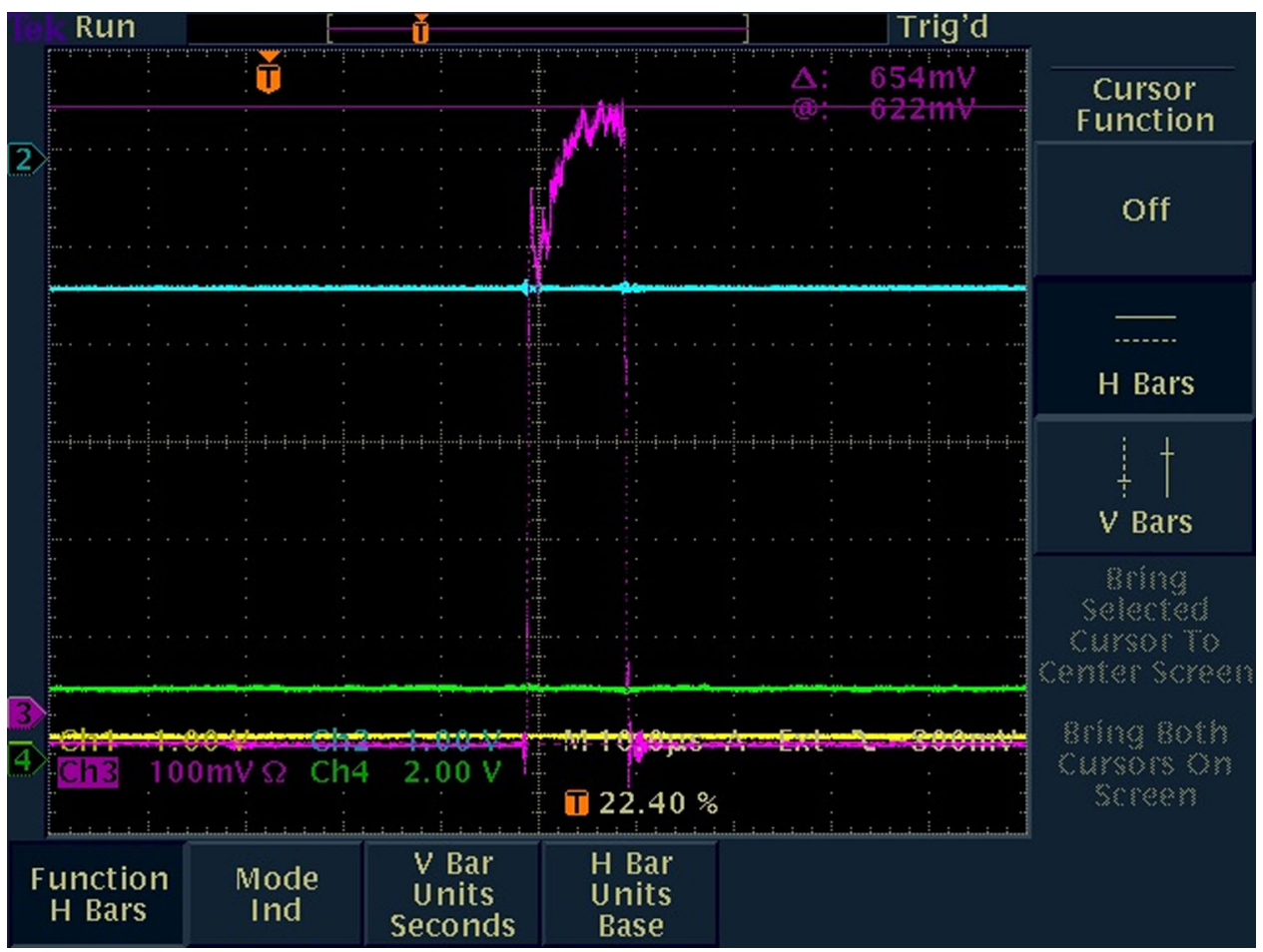

FIG. 33. This is an oscilloscope image of the $40 \mathrm{~mA}$ of peak current (magenta curve) measured on a toroid at the start of the DTL. This current was achieved on 25 Jun 2013 . The conversion between voltage and beam current is $(654 \mathrm{mV}) \times(0.0613 \mathrm{~mA} / \mathrm{mV})=40.1 \mathrm{~mA}$. 


\section{ACKNOWLEDGMENTS}

The successful testing, installing and commissioning of this injector could not have happened without the extraordinary dedication and work performed by the technicians, surveyors, engineers and scientists from FNAL, Goethe University Frankfurt, and LANL. The authors would like to thank the following people at FNAL who worked tirelessly during the entire test and installation phase of the RFQ injector: B. J. Ogert, J. C. Briney, and J. D. Kubinski for dis/assembling the entire RFQ injector many times, overall installation, and vacuum work; A. K. Triplett and J.F. Larson for the immense amount of vacuum work they did for the test stand and final installation; K. K. Koch and A. J. Feld for $\mathrm{H}^{-}$source work; M. J. Kucera, D. A. Arveson, E. A. Peoples-Evans, and R. Florian for controls work; G. R. Tassotto and the instrumentation group; B. Oshinowo, C. J. Wilson, and the survey group; S. L. Hays, M. A. Dilday, and B. A. Claypool for power supply work; R. Madrak for proofreading this manuscript. The authors would also like to thank colleagues at BNL who generously shared their expertise: J. Alessi, D. Raparia, V. Lodestro, and M. Okamura; at LANL, for useful discussions that helped clarify the effects of end-gap fields: L. J. Rybarcyk and R. W. Garnett; to the manufacturing company Firma Kress $\mathrm{GmbH}$, especially to J. Häuser for his great cooperation in this project. This work is supported by Fermi Research Alliance, LLC under Contract No. DE-AC0207CH11359 with the U.S. Department of Energy and the Bundesministerium für Bildung und Forschung (BMBF).

[1] M. Weiss, in Proceedings of EPAC 1992, p. 539, 1992.

[2] J. G. Alessi et al., in Proceedings of the 1987 Particle Accelerator Conference edited by E. R. Lindstrom and L. S. Taylor (IEEE, Washington, DC, 1987).

[3] A. P. Letchford et al., in Proceedings of the 21st Particle Accelerator Conference, Knoxville, TN, 2005 (IEEE, Piscataway, NJ, 2005).

[4] The original specifications did not have tolerances for the output energy or transverse emittances. A guide can be found by looking at the measurements performed on the beam at the exit of the C-Ws. It was found experimentally that the low energy acceptance of the first DTL is $735 \mathrm{keV}$ which is $2 \%$ below $750 \mathrm{keV}$. It is not possible to go above $750 \mathrm{keV}$ with the C-Ws and so an educated guess is that the energy acceptance is $\pm 2 \%$ of $750 \mathrm{keV}$ or $(750 \pm 15) \mathrm{keV}$.

[5] A. Schempp, in Proceedings of LINAC 1996, Geneva, Switzerland (1996), pp. 53-55.
[6] G. Romanov (private communication).

[7] Firma Kress GmbH. Biebergemünd Wirtheim, Germany, http://www.firma-kress.de.

[8] J. Schmidt, B. Koubek, and A. Schempp, in Proceedings of the 2nd International Particle Accelerator Conference, San Sebastiáán, Spain (EPS-AG, Spain, 2011), p. 2580.

[9] RFQ power in this paper is always defined to be (forward power-reverse power).

[10] http://techportal.eere.energy.gov/application.do/ID=2566.

[11] C. Y. Tan et al., Fermilab, Technical Report No. Beamsdoc-3646-v14, 2013.

[12] J. W. Staples, Lawrence Berkeley Laboratory, Berkeley Technical Report No. LBL-29472, 1990.

[13] I. M. Kapchinsky and V. A. Tepliakov, Prib. Tekh. Eksp. 2, 119 (1970).

[14] S. S. Kurennoy, R. W. Garnett, and L. J. Rybarcyk, in Proceedings of the 4th International Particle Accelerator Conference, IPAC-2013, Shanghai, China, 2013 (JACoW, Shanghai, China, 2013).

[15] M. Comunian and A. Pisent, in Proceedings of the 17th International Linear Accelerator Conference (LINAC1994), Tsukuba, Japan, 1994 (KEK, Tsukuba, Japan, 1994), p. 737.

[16] A. Pisent, in Design of the Heavy-Ion Injector PIAVE, edited by K. Shepard, AIP Conference Proceedings No. 473 (AIP, New York, 1998), p. 214.

[17] T. P. Wangler, RF Linear Accelerators (Wiley-VHC, New York, 2008), 2nd ed.

[18] K. R. Crandall, T. P. Wangler, L. M. Young, J. H. Billen, G. H. Neuschaefer, and D. L. Schrage, Los Alamos National Laboratories, Report No. LA-UR-96-1836, 2005.

[19] J. Schmidt, B. Koubek, and A. Schempp, in Proceedings of the 3rd International Particle Accelerator Conference, New Orleans, LA, 2012, edited by J. Corbett et al. (IEEE, Piscataway, NJ, 2012), p. 3818.

[20] B. Koubek, J. Schmidt, and A. Schempp, in Proceedings of the 3rd International Particle Accelerator Conference, New Orleans, LA, 2012 (IEEE, Piscataway, NJ, 2012), p. 3815.

[21] The shunt impedance $R_{P 0}$ like it is used in this paper is commonly used in the four-rod RFQ community. It refers to the peak voltage as shown above. In contrast to $R_{P}=\frac{U_{0}^{2}}{2 N}$ compares the effective voltage $U_{\text {eff }}^{2}=\frac{U_{0}^{2}}{2}$ to the average power.

[22] R. Gobin, P.-Y. Beauvais, R. Ferdinand, J. M. Lagniel, P. Ausset, B. Monsanglant, B. Pottin, and N. Rouvière, Vacuum 60, 261 (2001).

[23] J. Schmidt, B. Koubek, and A. Schmepp, in Proceedings of the 4th International Particle Accelerator Conference, IPAC-2013, Shanghai, China, 2013 (JACoW, Shanghai, China, 2013). 